\title{
Maximizers for the Strichartz norm for small solutions of mass-critical NLS
}

\author{
Thomas Duyckaerts, Frank Merle and Svetlana Roudenko
}

\begin{abstract}
Consider the mass-critical nonlinear Schrödinger equations in both focusing and defocusing cases for initial data in $L^{2}$ in space dimension $N$. By Strichartz inequality, solutions to the corresponding linear problem belong to a global $L^{p}$ space in the time and space variables, where $p=2+\frac{4}{N}$. In $1 D$ and $2 D$, the best constant for the Strichartz inequality was computed by D. Foschi who has also shown that the maximizers are the solutions with Gaussian initial data.

Solutions to the nonlinear problem with small initial data in $L^{2}$ are globally defined and belong to the same global $L^{p}$ space. In this work we show that the maximum of the $L^{p}$ norm is attained for a given small mass. In addition, in $1 D$ and $2 D$, we show that the maximizer is unique and obtain a precise estimate of the maximum. In order to prove this we show that the maximum for the linear problem in $1 D$ and $2 D$ is nondegenerated.
\end{abstract}

Mathematics Subject Classification (2010): 35Q55 (primary); 35P25, 35B50, 35B45 (secondary).

\section{Introduction}

We study the $L^{2}$-critical nonlinear Schrödinger (NLS) equation in space dimension $N \geq 1$ :

$$
\left\{\begin{array}{l}
i \partial_{t} u+\frac{1}{2} \Delta u+\gamma|u|^{\frac{4}{N}} u=0, \quad(t, x) \in \mathbb{R} \times \mathbb{R}^{N}, \\
u_{\mid t=0}=f \in L^{2}\left(\mathbb{R}^{N}\right) .
\end{array}\right.
$$

We will consider both focusing $(\gamma=+1)$ and defocusing $(\gamma=-1)$ equations.

Let us first recall some properties of the linear problem:

$$
i \partial_{t} u+\frac{1}{2} \Delta u=0, \quad u_{\uparrow t=0}=f
$$

This project was partially supported by the French ANR grant ONDNONLIN. S.R. was partially supported by the NSF grant DMS-0808081.

Received December 18, 2009; accepted April 20, 2010. 
Denote by $u=e^{i \frac{t}{2} \Delta} f$ the solution to (1.2). The mass $\|u(t)\|_{L^{2}}^{2}$ of the solution is conserved. Solutions to the linear problem satisfy the Strichartz inequality (see [24]):

$$
\forall f \in L^{2}, \quad\left\|e^{i \frac{t}{2} \Delta} f\right\|_{L_{t, x}^{\frac{4}{N}+2}} \leq C\|f\|_{L^{2}}
$$

where

$$
\|u\|_{L_{t, x}^{\frac{4}{N}+2}}=\left(\iint_{\mathbb{R} \times \mathbb{R}^{N}}|u(t, x)|^{\frac{4}{N}+2} d t d x\right)^{\frac{1}{\frac{4}{N}+2}} .
$$

By standard profile decomposition arguments, one can easily show that the maximum for the Strichartz inequality is attained. The best constant and maximizers for the Strichartz estimates were computed by D. Foschi [11] (see also [13] for another proof) for $N=1,2$. Before stating this result, we first recall some symmetries of the equations (1.1) and (1.2).

The following group of transformations leaves the solutions invariant under the nonlinear and linear Schrödinger evolution. If $\left\{\theta_{0}, \rho_{0}, t_{0}, \xi_{0}, x_{0}\right\} \in \mathbb{R} \times(0,+\infty) \times$ $\mathbb{R} \times \mathbb{R}^{N} \times \mathbb{R}^{N}$, then if $u$ is a solution to (1.1) (respectively (1.2)), so is

$$
e^{i \theta_{0}} \rho_{0}^{\frac{N}{2}} e^{i x \cdot \xi_{0}} e^{-i \frac{t}{2}\left|\xi_{0}\right|^{2}} u\left(\rho_{0}^{2} t+t_{0}, \rho_{0}\left(x-\frac{t}{2} \xi_{0}\right)+x_{0}\right) .
$$

This includes phase invariance, scaling, time-translation, Galilean transformation and space-translation. Another transformation of (1.1) and (1.2) is the pseudoconformal inversion (see [25]):

$$
\frac{1}{t^{N / 2}} \exp \left(\frac{i|x|^{2}}{2 t}\right) u\left(-\frac{1}{t}, \frac{x}{t}\right)
$$

Note that all the preceding transformations leave the mass and the $L_{t, x}^{\frac{4}{N}+2}$ norm of the solutions invariant. The linear equation is of course also invariant under the multiplication by a scalar: if $u(t, x)$ is a solution, so is $c_{0} u(t, x), c_{0} \in \mathbb{R}$.

Consider the following normalized Gaussian:

$$
G_{0}(x)=\frac{1}{\pi^{N / 4}} e^{-\frac{|x|^{2}}{2}}, \quad \text { thus, } \quad \int_{\mathbb{R}^{N}}\left|G_{0}\right|^{2} d x=1,
$$

and its linear evolution:

$$
G(t, x)=e^{\frac{1}{2} i t \Delta} G_{0}=\frac{1}{\pi^{N / 4}} \frac{1}{(1+i t)^{N / 2}} e^{-\frac{|x|^{2}}{2(1+i t)}} .
$$

Theorem A (Foschi). For all $f \in L^{2}\left(\mathbb{R}^{N}\right), N=1,2$,

$$
\left\|e^{i \frac{t}{2} \Delta} f\right\|_{L_{t, x}^{\frac{4}{N}+2}}^{\frac{4}{N}+2} \leq C_{S}\|f\|_{L^{2}\left(\mathbb{R}^{N}\right)}^{\frac{4}{N}+2}, \quad C_{S}= \begin{cases}\frac{1}{\sqrt{3}}, & N=1 \\ \frac{1}{2}, & N=2 .\end{cases}
$$


Furthermore, the equality holds if and only if $e^{i \frac{t}{2} \Delta} f$ is, up to the symmetries (1.4) of the equation, one of the solutions $c_{0} G, c_{0} \in \mathbb{C}$.

Let us mention that the effect of the pseudo-conformal transformation (1.5) on $G$ may be expressed only with the invariances (1.4) and we can omit it from consideration in Theorem A.

The Strichartz estimate (1.3) is the key ingredient to prove that the Cauchy problem (1.1) is locally wellposed in $L^{2}$ (see [8]). For small data, the solution is also globally wellposed and the global $L_{t, x}^{\frac{4}{N}+2}$ norm is finite, which implies that the solution scatters in $L^{2}$. This was extended to large radial data in the defocusing case $\gamma=-1$, in [30] for $N \geq 3$ and in [16] for $N=2$ (in this last work, the focusing case $\gamma=1$ below the mass of the ground-state is also treated). The proofs are mainly based on technics developed for the energy-critical NLS (see e.g. $[1,2,26,29]$ and $[15])$.

In all these studies, a global Strichartz norm (in the mass-critical case, the $L^{\frac{4}{N}+2}$ norm) appears as the relevant norm to control. In this work we consider

$$
I(\delta)=\sup _{\|f\|_{L^{2}\left(\mathbb{R}^{N}\right)}=\delta} \iint_{\mathbb{R} \times \mathbb{R}^{N}}|u(t, x)|^{\frac{4}{N}+2} d t d x,
$$

where $\delta>0$ is small and $u$ is the solution to (1.1). The results cited above imply that $I(\delta)$ is finite for small $\delta$, and, in the defocusing case with $N \geq 2$, for large $\delta$ if we restrict the maximum to radial solutions. A natural extension to Theorem A would be to show that this maximum is achieved by a unique solution (up to symmetries) of (1.1) and give a precise estimate of $I(\delta)$.

Our main result is the following:

Theorem 1.1. Fix $\gamma \in\{-1,+1\}$. There exists a $\delta_{0}>0$ such that for all $\delta$ in $\left(0, \delta_{0}\right)$, the maximum $I(\delta)$ is attained: there exists a solution $u_{\delta}$ of $(1.1)$ with initial condition $f_{\delta}$ such that

$$
\left\|f_{\delta}\right\|_{L^{2}}=\delta, \quad I(\delta)=\iint_{\mathbb{R} \times \mathbb{R}^{N}}\left|u_{\delta}(t, x)\right|^{\frac{4}{N}+2} d t d x .
$$

If $N=1$ or $N=2$, the maximizer $u_{\delta}$ is unique up to the transformations (1.4), (1.5) of the equation. Furthermore, as $\delta \rightarrow 0$,

$$
I(\delta)=C_{S} \delta^{\frac{4}{N}+2}+\gamma D_{N} \delta^{\frac{8}{N}+2}+\mathcal{O}\left(\delta^{\frac{12}{N}+2}\right),
$$

where $D_{1}=\frac{1}{\pi} \sum_{k \geq 1} \frac{(2 k) !}{k 9^{k}(k !)^{2}} \approx 0.0867$ and $D_{2}=\frac{1}{2 \pi} \ln \frac{4}{3} \approx 0.0458$.

Remark 1.2. In particular, in the focusing case in $1 D$ and $2 D$, the maximum of the Strichartz norm is, for small data, higher than in the linear case. In the defocusing case, the effect of the nonlinearity is to lower this maximum. 
Remark 1.3. The constant $D_{N}$ may be expressed as

$$
D_{N}=-\left(2+\frac{4}{N}\right) \operatorname{Im} \iint|G(t)|^{\frac{4}{N}} \bar{G}(t) \int_{0}^{t} e^{i \frac{(t-s)}{2} \Delta}\left(|G(s)|^{\frac{4}{N}} G(s)\right) d s d t d x
$$

Remark 1.4. The proof also shows that in $1 D$ and $2 D$, the initial condition of any maximizer with small mass $\delta$ is (after transformations) close to $\delta G_{0}$, where $G_{0}$ is the normalized Gaussian. See Proposition 3.3 and Remark 3.4 for a precise statement.

Estimates of Strichartz norms for critical nonlinear problems are only known in a few cases. Super-exponential bounds were obtained by T. Tao for radial defocusing energy-critical equations: Schrödinger equation in space dimension higher than 3 [26], and wave equation in $3 D$ [27]. An equivalent of the maximizum is given in [9] for the energy-critical focusing Schrödinger and wave equations (in space dimensions 3,4 and 5), close to the energy threshold given by the stationary solution.

The fact that the maximum of the Strichartz norm is attained is new for a nonlinear equation. The proof of this result is based on time-dependent adaptation to concentration-compactness arguments (see e.g. [18]) and on a super-additivity property of $I(\delta)$ which we show by general estimates on small solutions of (1.1). As stated in Proposition 2.12, the proof would extend to larger data provided the scattering of all solutions and the super-additivity properties are shown for those data also. This proof is flexible and should also easily adapt to other equations, e.g. the energy-critical NLS and wave equations for small data and (together with the methods of [9]) close to the energy threshold.

On the other hand, the proof of the uniqueness of the maximizer and of the estimate (1.7) is specific to the mass-critical problem, and strongly relies on the results of [11] and [13]. A key element is the nondegeneracy of the Gaussian for the nonlinear problem, in the orthogonal space of the null directions related to the invariances of the equation:

Theorem 1.5. Assume $N=1,2$. There exists $c>0$ such that if $\varphi \in L^{2}$ satisfies the following orthogonality properties $\left(x \in \mathbb{R}^{N}\right)$

$$
\int \varphi G_{0}=\int \varphi|x|^{2} G_{0}=0, \quad \int \varphi x G_{0}=0_{\mathbb{R}^{N}},
$$

then

$$
Q(\varphi) \geq c\|\varphi\|_{L^{2}}^{2},
$$

where $Q$ is the quadratic form associated to the second derivative of the mapping

$$
f \mapsto C_{S}\left(\int|f|^{2} d x\right)^{1+\frac{2}{N}}-\iint\left|e^{i \frac{t}{2} \Delta} f\right|^{2+\frac{4}{N}} d t d x
$$

from $L^{2}$ to $[0, \infty)$, at the critical point $f=G_{0}$. 
We refer to (3.3) for an expression of $Q$. This result is an analogue, for the Strichartz estimate, to the non-degeneracy of the maximizer $\frac{1}{\left(1+|x|^{2}\right)^{\frac{N-2}{2}}}$ for the Sobolev imbedding $\dot{H}^{1}\left(\mathbb{R}^{N}\right) \hookrightarrow L^{\frac{2 N}{N-2}}\left(\mathbb{R}^{N}\right)$ (see [21]).

To show Theorem 1.5, we apply a lens transform ([4, 20, 22]), related to the pseudo-conformal inversion, to the solutions of (1.1), which turns the Laplace operator into the harmonic operator $-\Delta+|x|^{2}$. The result then follows from explicit computations and a formula of Wei-Min Wang [33] on products of eigenfunctions for the harmonic oscillator.

The outline of the paper is as follows. In Section 2 we show that the maximizer is attained and in Section 3 we prove the estimate on $I(\delta)$. In Section 4 we show the uniqueness of the maximizer. Section 5 is devoted to the proof of Theorem 1.5.

ACKNOWLEDGEMENTS. The authors would like to thank Keith Rogers for pointing out the article [33]. Part of the project was done at the Institut Henri Poincaré in Paris during the special trimester Ondes non-linéaires et dispersion (April-July 2009).

\section{Existence of a maximizer}

In this section, where there is no restriction on the dimension $N \geq 1$, we show the first part of Theorem 1.1:

Proposition 2.1. There exists $\delta_{0}>0$ such that if $\delta \in\left(0, \delta_{0}\right)$, then there exists a solution $u_{\delta}$ of (1.1), with initial condition $f_{\delta}$ such that

$$
\left\|f_{\delta}\right\|_{L^{2}\left(\mathbb{R}^{N}\right)}=\delta \quad \text { and } \quad \iint_{\mathbb{R} \times \mathbb{R}^{N}}\left|u_{\delta}\right|^{\frac{4}{N}+2} d t d x=I(\delta) .
$$

After some preliminaries (Section 2.1) we show in Section 2.2 a crucial superadditivity property of $I(\delta)$, which relies on rough estimates of $I(\delta)$ and its growth rate. In Section 2.3 we use this property to prove Proposition 2.1 by concentrationcompactness arguments.

\subsection{Profile decomposition}

We recall here from [19] a profile decomposition adapted to the Strichartz estimate for the linear equation (1.2). We start with a long time perturbation result for the equation (1.1).

Lemma 2.2 (Long time perturbation). Let $A>0$. There exists $C=C(A)>0$ and a small $\delta_{0}=\delta_{0}(A)>0$ such that the following holds: Let $u \in C^{0}\left(\mathbb{R}, L_{x}^{2}\right)$ and solves

$$
i \partial_{t} u+\frac{1}{2} \Delta u+\gamma|u|^{\frac{4}{N}} u=0 .
$$


Let $\tilde{u}=\tilde{u}(x, t) \in C^{0}\left(\mathbb{R}, L_{x}^{2}\right)$ and define

$$
e=i \partial_{t} \tilde{u}+\frac{1}{2} \Delta \tilde{u}+\gamma|\tilde{u}|^{\frac{4}{N}} \tilde{u} .
$$

Assume $\|\tilde{u}\|_{L_{t, x}^{\frac{4}{N}+2}} \leq A$, and for some $\varepsilon<\delta_{0}$

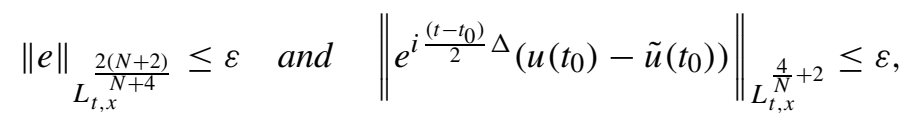

then

$$
\|u-\tilde{u}\|_{L_{t, x}^{\frac{4}{N}+2}} \leq C \varepsilon .
$$

We skip the proof of Lemma 2.2. We refer to $[2,6,15,29]$ for similar result for the energy-critical case, [12] for a subcritical case and [31, Lemma 3.1] for a statement close to Lemma 2.2 in the mass-critical case.

We next turn to the profile decomposition. If $\Gamma_{0}=\left\{\rho_{0}, t_{0}, \xi_{0}, x_{0}\right\} \in(0,+\infty) \times$ $\mathbb{R} \times \mathbb{R}^{N} \times \mathbb{R}^{N}$, and $u$ is a function of space and time, we will denote by $\Gamma_{0}(u)$ the function

$$
\Gamma_{0}(u)=\rho_{0}^{\frac{N}{2}} e^{i x \cdot \xi_{0}} e^{-i \frac{t}{2}\left|\xi_{0}\right|^{2}} u\left(\rho_{0}^{2} t+t_{0}, \rho_{0}\left(x-\frac{t}{2} \xi_{0}\right)+x_{0}\right) .
$$

As we have seen in the introduction, if $u$ is a solution to the linear equation (1.2) (respectively, to the nonlinear equation (1.1)), then $\Gamma_{0}(u)$ is also a solution to (1.2) (respectively, to (1.1)). We say that two sequences of transformations $\left\{\Gamma_{n}^{1}\right\}_{n}$ and $\left\{\Gamma_{n}^{2}\right\}_{n}$ are orthogonal when

$$
\lim _{n \rightarrow \infty} \frac{\rho_{n}^{1}}{\rho_{n}^{2}}+\frac{\rho_{n}^{2}}{\rho_{n}^{1}}+\frac{\left|\xi_{n}^{1}-\xi_{n}^{2}\right|}{\rho_{n}^{1}}+\left|t_{n}^{1}-t_{n}^{2}\right|+\left|\frac{t_{n}^{1}}{2} \frac{\xi_{n}^{1}-\xi_{n}^{2}}{\rho_{n}^{1}}+x_{n}^{1}-x_{n}^{2}\right|=+\infty .
$$

We recall from [19, Theorem 2] (see [14] in space dimension 1, [3] for general space dimension), the following profile decomposition result:

Lemma 2.3. Let $\left\{f_{n}\right\}$ be a bounded sequence in $L^{2}\left(\mathbb{R}^{N}\right)$. Then there exists a subsequence of $\left\{f_{n}\right\}$ (still denoted by $\left\{f_{n}\right\}$ ), a family $\left\{U^{j}\right\}_{j \geq 1}$ of solutions to (1.2), and sequences of parameters $\left\{\Gamma_{n}^{j}\right\}_{n}$, such that if $j \neq k,\left\{\Gamma_{n}^{j}\right\}_{n}$ is orthogonal to $\left\{\Gamma_{n}^{k}\right\}_{n}$ and for all $\mathrm{J}$,

$$
f_{n}(x)=\sum_{j=1}^{J} \Gamma_{n}^{j}\left(U^{j}\right)(0, x)+h_{n}^{J}(x)
$$

where

$$
\lim _{J \rightarrow+\infty} \limsup _{n \rightarrow \infty}\left\|e^{i \frac{t}{2} \Delta} h_{n}^{J}\right\|_{L_{t, x}^{\frac{4}{N}+2}}=0
$$


Remark 2.4. As a consequence of the orthogonality of the transformations $\Gamma_{n}^{j}$, the following Pythagorean expansions hold for all $J \geq 1$ :

$$
\begin{array}{r}
\left\|f_{n}\right\|_{L^{2}}^{2}-\sum_{j=1}^{J}\left\|U^{j}(0)\right\|_{L^{2}}^{2}-\left\|h_{n}^{J}\right\|_{L^{2}}^{2} \underset{n \rightarrow+\infty}{\longrightarrow} 0, \\
\left\|e^{i \frac{t}{2} \Delta} f_{n}\right\|_{L_{t, x}^{\frac{4}{N}+2}}^{\frac{4}{N}+2}-\sum_{j=1}^{J}\left\|U^{j}\right\|_{L_{t, x}^{\frac{4}{N}+2}}^{\frac{4}{N}+2}-\left\|e^{i \frac{t}{2} \Delta} h_{n}^{J}\right\|_{L_{t, x}^{\frac{4}{N}+2} \underset{n \rightarrow+\infty}{\longrightarrow}}^{\longrightarrow} 0 .
\end{array}
$$

Let $\left\{f_{n}\right\}_{n}$ be a sequence in $L^{2}$ and assume that the corresponding solution to (1.1) is globally defined and satisfies $\left\|f_{n}\right\|_{L_{t, x}^{\frac{4}{N}+2}}<\infty$. Consider the profile decomposition given by Lemma 2.3. Let $V^{j}$ be the nonlinear profile associated to $\left\{U^{j}, t_{n}^{j}\right\}_{n}$, that is the unique solution of (1.1) such that

$$
\lim _{n \rightarrow \infty}\left\|U^{j}\left(t_{n}^{j}\right)-V^{j}\left(t_{n}^{j}\right)\right\|_{L^{2}}=0
$$

Assume also that the $V^{j}$ 's are globally defined and such that $\left\|V^{j}\right\|_{L^{2+\frac{4}{N}}}$ is finite for all $j$. Combining Lemmas 2.2 and 2.3, one gets a nonlinear version of the decomposition (2.4):

Corollary 2.5. Let $\left\{f_{n}\right\}_{n}$ is as above and $\left\{u_{n}\right\}_{n}$ be the sequence of solutions to (1.1) with initial conditions $\left\{f_{n}\right\}_{n}$. Then

$$
u_{n}(t, x)=\sum_{j=1}^{J} \Gamma_{n}^{j}\left(V^{j}\right)(t, x)+h_{n}^{J}(t, x)+r_{n}^{J}(t, x)
$$

with

$$
\lim _{J \rightarrow+\infty} \lim _{n \rightarrow+\infty}\left(\left\|r_{n}^{J}\right\|_{L_{t, x}^{\frac{4}{N}+2}}+\sup _{t \in \mathbb{R}}\left\|r_{n}^{J}(t)\right\|_{L^{2}}\right)=0
$$

Remark 2.6. Using the orthogonality of the sequences of transformations $\left\{\Gamma_{n}^{j}\right\}_{n}$, it is easy to check that

$$
\lim _{J \rightarrow \infty} \limsup _{n \rightarrow \infty}\left|\left\|u_{n}\right\|_{L_{t, x}^{\frac{4}{N}+2}}^{\frac{4}{N}+2}-\sum_{j=1}^{J} \| V^{j}\right| \begin{gathered}
\frac{4}{N}+2 \\
\frac{4}{N}+2
\end{gathered} \mid=0 .
$$

\subsection{A superadditivity property of the maximum}

In this paragraph we give various estimates on $I(\delta)$. The main result is the following proposition, which is one of the steps (along with a concentration-compactness argument) in showing that the maximizer is attained: 
Proposition 2.7. There exists $\delta_{0}>0$ such that if $0<\sqrt{\alpha^{2}+\beta^{2}}<\delta_{0}$, then

$$
I(\alpha)+I(\beta)<I\left(\sqrt{\alpha^{2}+\beta^{2}}\right) .
$$

Remark 2.8. Superadditivity (or subadditivity for minimizers) conditions are classical in this context (see [17, Subsection I.2]).

The proof of Proposition 2.7 relies on two estimates on $I(\delta)$ that we treat in Lemmas 2.9 and 2.11 below.

Lemma 2.9. There exists a constant $C_{0}>0$ such that for small $\delta>0$,

$$
\left|I(\delta)-C_{S} \delta^{\frac{4}{N}+2}\right| \leq C_{0} \delta^{\frac{8}{N}+2},
$$

where $C_{S}$ is the best constant for the Strichartz inequality

$$
\iint\left|e^{i \frac{t}{2} \Delta} f\right|^{\frac{4}{N}+2} d t d x \leq C_{S}\|f\|_{L^{2}}^{\frac{4}{N}+2} .
$$

Before proving this lemma, we start by a straightforward consequence of the small data well-posedness theory for equation (1.1) (see [8]).

Claim 2.10. There exists a constant $C>0$ such that if $\|f\|_{L^{2}}$ is small, then

$$
\left\|e^{i \frac{t}{2} \Delta} f-u\right\|_{L_{t, x}^{\frac{4}{N}+2}} \leq C\|f\|_{L^{2}}^{\frac{4}{N}+1}
$$

where $u$ is the solution of (1.1) with initial condition $f$.

Sketch of proof. The Cauchy problem theory for (1.1) implies that for small initial data

$$
\|u\|_{L_{t, x}^{\frac{4}{N}+2}} \leq 2\|f\|_{L^{2}} .
$$

Since

$$
u(t)=e^{i \frac{t}{2} \Delta} f+i \gamma \int_{0}^{t} e^{\frac{i}{2}(t-s) \Delta}|u(s)|^{\frac{4}{N}} u(s) d s,
$$

the claim follows from Theorem A and the Strichartz estimate

$$
\left\|\int_{0}^{t} e^{\frac{i}{2}(t-s) \Delta} \varphi(s) d s\right\|_{L_{t, x}^{\frac{4}{N}+2}} \leq C\|\varphi\|_{L_{t, x}^{\frac{2(N+2)}{N+4}}} .
$$


Proof of Lemma 2.9. Let $u$ be a solution of (1.1) with initial condition $f$ such that $\|f\|_{L^{2}\left(\mathbb{R}^{N}\right)}=\delta$. Then

$$
\begin{aligned}
& \left.\left|\iint\right| e^{i \frac{t}{2} \Delta} f(x)\right|^{\frac{4}{N}+2} d t d x-\iint|u(t, x)|^{\frac{4}{N}+2} d t d x \mid \\
& \leq C \mid\left\|e^{i \frac{t}{2} \Delta} f\right\|_{L_{t, x}^{\frac{4}{N}+2}-\|u\|_{L_{t, x}^{\frac{4}{N}+2}} \mid\left(\left\|e^{i \frac{t}{2} \Delta} f\right\|_{L_{t, x}^{\frac{4}{N}+2}}^{\frac{4}{N}+1}\|u\|_{L_{t, x}^{\frac{4}{N}+2}}^{\frac{4}{N}+1}\right)} \\
& \leq C\left\|e^{i \frac{t}{2} \Delta} f-u\right\|_{L_{t, x}^{\frac{4}{N}+2}} \delta^{\frac{4}{N}+1} \leq C \delta^{\frac{8}{N}+2}
\end{aligned}
$$

where the last line follows from the triangle inequality and then from Claim 2.10. Applying the previous inequality to the initial data $f=\delta F_{0}$, where $F_{0}$ is the initial condition of a maximizer for Strichartz estimate (2.10), and then to a sequence $\left\{f_{n}\right\}_{n}$ such that $\left\|f_{n}\right\|_{L^{2}}=\delta$ and $\iint\left|u_{n}\right|^{\frac{4}{N}+2} \rightarrow I(\delta)$, we obtain (2.9).

We next estimate the rate of growth of $I(\delta)$.

Lemma 2.11. If $\delta$ is small and $\varepsilon \leq \frac{1}{2} \delta$, then

$$
I(\delta)+c_{1} \delta^{\frac{4}{N}+1} \varepsilon \leq I(\delta+\varepsilon) \leq I(\delta)+C_{1} \delta^{\frac{4}{N}+1} \varepsilon,
$$

where $c_{1}=\frac{4}{N} C_{S}$ and $C_{1}=2\left(\frac{4}{N}+2\right) C_{S}$.

Proof. Step 1. We first show that there exist $C_{2}, \epsilon_{0}>0$ such that if $f \in L^{2}$ with $\|f\|_{L^{2}}+\epsilon \leq \epsilon_{0}, u$ is the solution of (1.1) with the initial condition $f$, and $v_{\epsilon}$ is the solution of (1.1) with the initial condition $(1+\epsilon) f$, then

$$
\left.\left|(1+\epsilon)^{\frac{4}{N}+2} \iint\right| u\right|^{\frac{4}{N}+2}-\iint\left|v_{\epsilon}\right|^{\frac{4}{N}+2} \mid \leq C_{2} \epsilon\|f\|_{L^{2}}^{\frac{8}{N}+2} .
$$

First, observe that $u_{\epsilon}=(1+\epsilon) u$ is a solution to the equation

$$
i \partial_{t} u_{\epsilon}+\frac{1}{2} \Delta u_{\epsilon}+\frac{1}{(1+\epsilon)^{\frac{4}{N}}}\left|u_{\epsilon}\right|^{\frac{4}{N}} u_{\epsilon}=0, \quad u_{\epsilon \mid t=0}=(1+\epsilon) f .
$$

We rewrite the above equation as

$$
i \partial_{t} u_{\epsilon}+\frac{1}{2} \Delta u_{\epsilon}+\left|u_{\epsilon}\right|^{\frac{4}{N}} u_{\epsilon}=\left(1-\frac{1}{(1+\epsilon)^{\frac{4}{N}}}\right)\left|u_{\epsilon}\right|^{\frac{4}{N}} u_{\epsilon},
$$

noting that for small $\epsilon$, Strichartz estimate implies

$$
\begin{gathered}
\left\|\left(1-\frac{1}{(1+\epsilon)^{\frac{4}{N}}}\right)\left|u_{\epsilon}\right|^{\frac{4}{N}} u_{\epsilon}\right\|{ }_{L_{t, x}} \frac{2(N+2)}{N+4} \leq C \epsilon\left\|\left|u_{\epsilon}\right|^{1+\frac{4}{N}}\right\|_{L_{t, x}} \leq \frac{2(N+2)}{N+4} \\
=C \epsilon\left\|u_{\epsilon}\right\|_{L_{t, x}^{\frac{4}{N}+2}}^{1+\frac{4}{N}} \leq C \epsilon\|f\|_{L^{2}}^{1+\frac{4}{N}} .
\end{gathered}
$$


Since $v_{\epsilon}$ is a solution of

$$
i \partial_{t} v_{\epsilon}+\frac{1}{2} \Delta v_{\epsilon}+\left|v_{\epsilon}\right|^{\frac{4}{N}} v_{\epsilon}=0, \quad v_{\epsilon \mid t=0}=(1+\epsilon) f
$$

by the long time perturbation Lemma 2.2, we get

$$
\left\|u_{\epsilon}-v_{\epsilon}\right\|_{L_{t, x}}^{\frac{4}{N}+2} \leq C \epsilon\|f\|_{L^{2}}^{\frac{4}{N}+1}
$$

Hence,

$$
\begin{aligned}
\left.\left|\iint\right| u_{\epsilon}\right|^{\frac{4}{N}+2} d t d x-\iint\left|v_{\epsilon}\right|^{\frac{4}{N}+2} d t d x \mid & \leq C\left\|u_{\epsilon}-v_{\epsilon}\right\|_{L_{t, x}^{\frac{4}{N}+2}}\|f\|_{L^{2}}^{\frac{4}{N}+1} \\
& \leq C \epsilon\|f\|_{L^{2}}^{\frac{8}{N}+2}
\end{aligned}
$$

which concludes Step 1.

Step 2. Let $\varepsilon, \delta>0$. First, we show the lower bound of $I(\delta+\varepsilon)$. Let $f \in L^{2}\left(\mathbb{R}^{N}\right)$ be such that

$$
\|f\|_{L^{2}}=\delta \quad \text { and } \quad \iint|u(t, x)|^{\frac{4}{N}+2} d t d x \geq I(\delta)-\delta^{\frac{8}{N}+1} \varepsilon,
$$

where $u$ is the corresponding solution of (1.1) and we used the supremum property of $I(\delta)$. Let $u_{\varepsilon}$ be the solution of (1.1) with the initial condition $\left(1+\frac{\varepsilon}{\delta}\right) f$. Then $\left\|u_{\varepsilon}(0)\right\|_{L^{2}}=\delta+\varepsilon$. By Step 1,

$$
\begin{aligned}
I(\delta+\varepsilon) & \geq \iint\left|u_{\varepsilon}(t, x)\right|^{\frac{4}{N}+2} d t d x \\
& \geq\left(1+\frac{\varepsilon}{\delta}\right)^{\frac{4}{N}+2} \iint|u(t, x)|^{\frac{4}{N}+2}-C_{2} \frac{\varepsilon}{\delta} \delta^{\frac{8}{N}+2} .
\end{aligned}
$$

By (2.12), we get

$$
I(\delta+\varepsilon) \geq\left[1+\left(\frac{4}{N}+2\right) \frac{\varepsilon}{\delta}\right]\left(I(\delta)-\delta^{\frac{8}{N}+1} \varepsilon\right)-C_{2} \delta^{\frac{8}{N}+1} \varepsilon .
$$

Lemma 2.9 implies $I(\delta) \geq C_{S} \delta^{\frac{4}{N}+2}-C_{0} \delta^{\frac{8}{N}+2}$, hence,

$$
\begin{aligned}
I(\delta+\varepsilon) \geq & I(\delta)+C_{S}\left(\frac{4}{N}+2\right) \delta^{\frac{4}{N}+1} \varepsilon \\
& -\left[\left(\frac{4}{N}+2\right) C_{0}+\left(1+\left(\frac{4}{N}+2\right) \frac{\varepsilon}{\delta}\right)+C_{2}\right] \delta^{\frac{8}{N}+1} \varepsilon .
\end{aligned}
$$


Now if $\varepsilon<\frac{1}{2} \delta$ and

$$
\delta<\left(\frac{C_{S}}{4+6 C_{0}+C_{2}}\right)^{N / 4},
$$

the last term in the expression above will be less than $2 C_{S} \delta^{\frac{4}{N}+1} \varepsilon$, and thus, the right side in (2.11) follows with $c_{1}=\frac{4}{N} C_{S}$.

The upper bound on $I(\delta+\varepsilon)$ follows similarly from Step 1 and Lemma 2.9, obtaining the left side in (2.11) with $C_{1}=2 C_{S}\left(\frac{4}{N}+2\right)$.

We next prove Proposition 2.7.

Proof. Without loss of generality, we can assume $0<\alpha \leq \beta$.

Step 1. We first show that there exists a large constant $C_{3}>0$ such that the conclusion of the proposition holds if

$$
C_{3} \beta^{\frac{2}{N}+1} \leq \alpha \leq \beta .
$$

By Lemma 2.9,

$$
\begin{aligned}
I(\alpha)+I(\beta) & \leq C_{S} \alpha^{\frac{4}{N}+2}+C_{S} \beta^{\frac{4}{N}+2}+2 C_{0} \beta^{\frac{8}{N}+2}, \\
\text { and } \quad C_{S}\left(\alpha^{2}+\beta^{2}\right)^{\frac{2}{N}+1} & \leq I\left(\sqrt{\alpha^{2}+\beta^{2}}\right)+2 C_{0} \beta^{\frac{8}{N}+2} .
\end{aligned}
$$

There is a constant $\kappa_{N}>0$ such that $1+x^{\frac{2}{N}+1}+\kappa_{N} x \leq(1+x)^{\frac{2}{N}+1}$ for $x \in[0,1]$. As a consequence, $\alpha^{\frac{4}{N}+2}+\beta^{\frac{4}{N}+2}+\kappa_{N} \beta^{\frac{4}{N}} \alpha^{2} \leq\left(\alpha^{2}+\beta^{2}\right)^{\frac{2}{N}+1}$. Combining with the previous estimates, we get

$$
I(\alpha)+I(\beta)+C_{S} \kappa_{N} \beta^{\frac{4}{N}} \alpha^{2}-4 C_{0} \beta^{\frac{8}{N}+2} \leq I\left(\sqrt{\alpha^{2}+\beta^{2}}\right),
$$

which yields the announced result if $C_{3}$ is chosen large in (2.13).

Step 2. We next show that the conclusion of the Proposition still holds if

$$
0<\alpha<C_{3} \beta^{\frac{2}{N}+1},
$$

where $C_{3}$ is the constant defined in Step 1 . Choosing $\delta_{0}$ small enough, $\beta \leq \delta_{0}$ and (2.14) imply

$$
\frac{\alpha^{2}}{4 \beta} \leq \sqrt{\alpha^{2}+\beta^{2}}-\beta \leq \frac{\beta}{2} .
$$

By Lemma 2.11, with $\delta=\beta$ and $\varepsilon=\sqrt{\alpha^{2}+\beta^{2}}-\beta$,

$$
I(\beta) \leq I\left(\sqrt{\alpha^{2}+\beta^{2}}\right)-c_{1} \beta^{\frac{4}{N}+1}\left(\sqrt{\alpha^{2}+\beta^{2}}-\beta\right) \leq I\left(\sqrt{\alpha^{2}+\beta^{2}}\right)-\frac{c_{1}}{4} \beta^{\frac{4}{N}} \alpha^{2} .
$$


Combining with Lemma 2.9 we get, taking a smaller $\delta_{0}$ if necessary,

$$
\begin{aligned}
I(\alpha)+I(\beta) & \leq I\left(\sqrt{\alpha^{2}+\beta^{2}}\right)-\frac{c_{1}}{4} \beta^{\frac{4}{N}} \alpha^{2}+2 C_{S} \alpha^{\frac{4}{N}+2} \\
& \leq I\left(\sqrt{\alpha^{2}+\beta^{2}}\right)+\alpha^{2} \beta^{\frac{4}{N}}\left(2 C_{S} C_{3}^{\frac{4}{N}} \beta^{\frac{8}{N^{2}}}-\frac{c_{1}}{4}\right)
\end{aligned}
$$

which shows that the conclusion of the proposition holds also in this case, provided $\delta_{0}>0$ is small enough.

\subsection{Proof of the existence of the maximizer}

Let us show Proposition 2.1. We will prove the following more general result:

Proposition 2.12. Assume that there exists a constant $A>0$ such that

i. Scattering: for all $f \in L^{2}$ such that $\|f\|_{L^{2}} \leq A$, the solution $u$ of (1.1) with initial condition $f$ is globally defined and

$$
\delta \leq A \Longrightarrow I(\delta)<\infty .
$$

ii. Superadditivity: if $0<\sqrt{\alpha^{2}+\beta^{2}}=A$, and $\alpha, \beta>0$, then

$$
I(\alpha)+I(\beta)<I(A) .
$$

Then there exists a solution $u_{A}$ of (1.1) with initial condition $f_{A} \in L^{2}$ such that

$$
\left\|f_{A}\right\|_{L^{2}}=A, \quad \iint\left|u_{A}\right|^{2+\frac{4}{N}}=I(A) .
$$

In view of the small data global well-posedness theory and Proposition 2.7, Proposition 2.12 implies Proposition 2.1. Let us prove Proposition 2.12.

Let $\left\{u_{n}\right\}_{n}$ be a sequence of solutions to (1.1) with initial data $f_{n}$ such that

$$
\left\|f_{n}\right\|_{L^{2}\left(\mathbb{R}^{N}\right)}=A, \quad \lim _{n \rightarrow \infty} \iint_{\mathbb{R}^{N}}\left|u_{n}\right|^{\frac{4}{N}+2}=I(A) .
$$

We will show that there exist a subsequence of $\left\{u_{n}\right\}_{n}$ and a sequence $\left\{\Gamma_{n}\right\}_{n}$ of transformations such that $\left\{\Gamma_{n}\left(u_{n}\right)\right\}_{n}$ converges strongly in $L^{2}$. Consider, after extraction, a profile decomposition of the sequence $\left\{f_{n}\right\}_{n}$ :

$$
f_{n}=\sum_{j=1}^{J} \Gamma_{n}^{j}\left(U^{j}\right)_{\lceil t=0}+h_{n}^{J}
$$

It is sufficient to show that $U^{j}=0$ except for one $j$ and that $\lim _{n \rightarrow \infty}\left\|h_{n}^{J}\right\|_{L^{2}}=0$, which we will do in two steps. 
Step 1: no dichotomy. First assume that there are at least two nonzero profiles, say $U^{1} \neq 0$ and $U^{2} \neq 0$. Let $V^{1}$ be the nonlinear profiles associated to $\left\{U^{1}, t_{n}^{1}\right\}$ and $V_{n}$ the solution of (1.1) given by

$$
V_{n}=\Gamma_{n}^{1}\left(V^{1}\right)
$$

Let $W_{n}$ be the sequence of solutions to (1.1) with initial condition

$$
W_{n}(0)=f_{n}-V_{n}(0)
$$

Let $r_{n}=u_{n}-V_{n}-W_{n}$. By assumption (2.12), all the nonlinear profiles $V^{j}$ scatter. Thus, one can use Corollary 2.5 , showing

$$
\lim _{n \rightarrow \infty} \sup _{t \in \mathbb{R}}\left\|r_{n}(t)\right\|_{L^{2}}=0
$$

Furthermore, (see (2.5) and Remark 2.6)

$$
\begin{aligned}
\int\left|f_{n}\right|^{2} & =\int\left|V_{n}(0)\right|^{2}+\int\left|W_{n}(0)\right|^{2}+o_{n}(1) \\
\iint\left|u_{n}\right|^{\frac{4}{N}}+2 & =\iint\left|V_{n}\right|^{\frac{4}{N}+2}+\iint\left|W_{n}\right|^{\frac{4}{N}+2}+o_{n}(1) .
\end{aligned}
$$

Let $\varepsilon=\left\|U^{1}(0)\right\|_{L^{2}}$. Then for all $n, \varepsilon=\left\|V_{n}(0)\right\|_{L^{2}}$. By (2.16),

$$
\left\|W_{n}(0)\right\|_{L^{2}}^{2}=A^{2}-\varepsilon^{2}+o_{n}(1) .
$$

By our assumptions, $\varepsilon>0$ (otherwise, $U^{1}$ would be zero) and $A^{2}-\varepsilon^{2}>0$ (otherwise, $U^{2}$ would be zero). Using that $\iint\left|u_{n}\right|^{\frac{4}{N}+2}$ tends to $I(A)$ as $n \rightarrow \infty$, and that by Lemma 2.2, $\lim \sup _{n} \int\left|W_{n}\right|^{\frac{4}{N}+2} \leq I\left(\sqrt{A^{2}-\varepsilon^{2}}\right)$, we get by (2.17)

$$
I(A) \leq I(\varepsilon)+I\left(\sqrt{A^{2}-\varepsilon^{2}}\right) .
$$

This contradicts assumption (2.12), concluding Step 1.

Step 2: non vanishing and the end of the proof. There must be one nonzero profile in (2.15). If not, then

$$
\lim _{n \rightarrow \infty} \iint\left|u_{n}\right|^{\frac{4}{N}+2}=0,
$$

showing that $I(A)=0$, a contradiction. It remains to show that the remainder $h_{n}=h_{n}^{J}$ in (2.15) tends to 0 in $L^{2}$. Denote by

$$
\varepsilon=\lim _{n \rightarrow \infty}\left\|h_{n}\right\|_{L^{2}}
$$

then, using again Lemma 2.2, we get $I(A) \leq I\left(\sqrt{A^{2}-\varepsilon^{2}}\right)$, which shows by assumption (2.12) that $\varepsilon=0$. 
Denoting by $U^{1}$ the only nonzero profile in (2.15), we have shown that $\left(\Gamma_{n}^{1}\right)^{-1}\left(u_{n}\right)$ tends to $U^{1}$ in $L^{2}$, and therefore,

$$
\left\|U^{1}\right\|_{L^{2}}=A, \quad \iint\left|U^{1}\right|^{\frac{4}{N}+2}=I(A),
$$

concluding the proof of the proposition.

\section{Estimate of the maximum of the Strichartz norm}

In the remainder of the paper, we restrict ourselves to $1 D$ and $2 D$. In this section we prove the second part of Theorem 1.1:

Proposition 3.1. Assume that $N=1$ or $N=2$. Then as $\delta \rightarrow 0$,

$$
I(\delta)=\iint\left|u_{\delta}\right|^{2+\frac{4}{N}}=C_{S} \delta^{2+\frac{4}{N}}+\gamma D_{N} \delta^{2+\frac{8}{N}}+\mathcal{O}\left(\delta^{2+\frac{12}{N}}\right),
$$

where $D_{1}=\frac{1}{\pi} \sum_{k \geq 1} \frac{(2 k) !}{k 9^{k}(k !)^{2}} \approx 0.0867$ and $D_{2}=\frac{1}{2 \pi} \ln \frac{4}{3} \approx 0.0458$.

Before proving Proposition 3.1, we define the quadratic form associated to the maximum of the Strichartz estimate that appears in Theorem 1.5. By Theorem A, if $G$ is the Gaussian solution defined by (1.6) and $\varphi \in L^{2}$, then

$$
C_{S}\left(\int\left|G_{0}+\varphi\right|^{2}\right)^{1+\frac{2}{N}}-\iint\left|G+e^{i \frac{t}{2} \Delta} \varphi\right|^{2+\frac{4}{N}} \geq 0 .
$$

Expanding the above inequality and using that $G$ is a maximizer, we obtain that the linear part vanishes, i.e.,

$$
\forall \varphi \in L^{2}, \quad C_{S} \operatorname{Re} \int G_{0} \varphi=\operatorname{Re} \iint|G|^{\frac{4}{N}} \bar{G} e^{i \frac{t}{2} \Delta} \varphi
$$

The expansion at second order in $\varphi$ yields

$$
C_{S}\left(\int\left|G_{0}+\varphi\right|^{2}\right)^{1+\frac{2}{N}}-\iint\left|G+e^{i \frac{t}{2} \Delta} \varphi\right|^{2+\frac{4}{N}}=Q(\varphi)+\mathcal{O}\left(\|\varphi\|_{L^{2}}^{3}\right),
$$

where $Q$ is a (real) nonnegative symmetric quadratic form on $L^{2}$ defined by

$$
\begin{aligned}
Q(\varphi)= & C_{S}\left[\frac{N+2}{N} \int|\varphi|^{2}+\frac{4(N+2)}{N^{2}}\left(\operatorname{Re} \int G_{0} \varphi\right)^{2}\right] \\
& -\frac{(N+2)^{2}}{N^{2}} \iint|G|^{\frac{4}{N}}\left|e^{i \frac{t}{2} \Delta} \varphi\right|^{2} \\
& -\frac{2(N+2)}{N^{2}} \operatorname{Re} \iint|G|^{\frac{4}{N}-2} \bar{G}^{2}\left(e^{i \frac{t}{2} \Delta} \varphi\right)^{2} .
\end{aligned}
$$


By the transformations of the linear equation (respectively, multiplication by a real number, phase shift, space translation, Galilean invariance, scaling and time translation), we have

$$
Q\left(G_{0}\right)=Q\left(i G_{0}\right)=Q\left(x G_{0}\right)=Q\left(i x G_{0}\right)=Q\left(x^{2} G_{0}\right)=Q\left(i x^{2} G_{0}\right)=0,
$$

if $N=1$ and

$$
Q\left(G_{0}\right)=Q\left(i G_{0}\right)=Q\left(x_{j} G_{0}\right)=Q\left(i x_{j} G_{0}\right)=Q\left(|x|^{2} G_{0}\right)=Q\left(i|x|^{2} G_{0}\right)=0,
$$

(where $j=1,2$ ) if $N=2$. Theorem 1.5, which will be proved in Section 5 states that $Q$ is positive definite in the subspace of functions in $L^{2}$ that are orthogonal to the directions in (3.4) or (3.5). This non-degeneracy property is crucial in the proof of Proposition 3.1, which is divided in two parts.

\subsection{Choice of the maximizer}

We first give a corollary to the linear profile decomposition that will be needed in the proof. Recall from (1.6) the definition of the normalized Gaussian $G$.

Lemma 3.2. Let $\left\{f_{n}\right\}_{n}$ be a sequence in $L^{2}\left(\mathbb{R}^{N}\right)$ such that

$$
\lim _{n \rightarrow \infty}\left\|f_{n}\right\|_{L^{2}}=1
$$

and

$$
\lim _{n \rightarrow \infty} \iint\left|e^{i \frac{t}{2} \Delta} f_{n}\right|^{\frac{4}{N}+2} d t d x=C_{S} .
$$

Then there exist a subsequence of $\left\{f_{n}\right\}_{n}$ (still denoted by $\left\{f_{n}\right\}_{n}$ ), a phase $\theta_{0}$ and a sequence $\left\{\Gamma_{n}\right\}_{n}$ of transformations of the form (2.2) such that

$$
\lim _{n \rightarrow \infty}\left\|f_{n}-e^{i \theta_{0}} \Gamma_{n}(G)\right\|_{L^{2}}=0,
$$

where $G$ is the normalized Gaussian solution defined in (1.6).

Proof. This is an application of Lemma 2.3 and the uniqueness result of Foschi [11].

After extraction of a subsequence, the sequence $\left\{f_{n}\right\}_{n}$ admits a profile decomposition of the form (2.4). At least one of the profiles is nonzero. Indeed, if it was not the case, $\left\|e^{i \frac{t}{2} \Delta} f_{n}\right\|_{L \frac{4}{N}+2}$ would tend to 0 , a contradiction with (3.7). Reordering the profiles, we may assume that $U^{1} \neq 0$. By the Pythagorean expansion (2.6) and by (3.7)

$$
C_{S}+o_{n}(1)=\iint\left|e^{i \frac{t}{2} \Delta} f_{n}\right|^{\frac{4}{N}+2} d t d x \leq C_{S}\left(\left\|U^{1}\right\|_{L^{2}}^{\frac{4}{N}+2}+\left\|w_{n}^{1}\right\|_{L^{2}}^{\frac{4}{N}+2}\right)+o_{n}(1) .
$$


Using that by (2.5), $\left\|w_{n}^{1}\right\|_{L^{2}}^{2}=1-\left\|U^{1}\right\|_{L^{2}}^{2}+o_{n}(1)$, we obtain from the previous expression that

$$
1 \leq\left(\left\|U^{1}\right\|_{L^{2}}^{2}\right)^{\frac{4}{N}+2}+\left(1-\left\|U^{1}\right\|_{L^{2}}^{2}\right)^{\frac{4}{N}+2}
$$

which shows that $\left\|U^{1}\right\|_{L^{2}}=1$ (we already excluded the case $\left\|U^{1}\right\|_{L^{2}}=0$ ), and by (2.5) again

$$
\lim _{n \rightarrow \infty}\left\|f_{n}-\Gamma_{n}^{1}\left(U^{1}\right)(0)\right\|_{L^{2}}=0 .
$$

By our assumptions on $f_{n}$ we obtain, passing to the limit, that $\left\|U^{1}(0)\right\|_{L^{2}}=1$ and $\left\|U^{1}\right\|_{L^{\frac{4}{N}+2}}^{\frac{4}{N}+2}=C_{S}$, which shows by Theorem A that $U^{1}(0)=G_{0}$ up to the symmetries of the equation (i.e., the transformations of the form (2.2) and the multiplication by a phase $e^{i \theta_{0}}$ ), which completes the proof.

Proposition 3.3. There exists $\delta_{0}>0$ such that if $\left\{u_{\delta}^{*}\right\}_{0<\delta<\delta_{0}}$ is a family of maximizers, i.e. $u_{\delta}^{*}$ satisfies $(2.1)$, then for all $\delta \in\left(0, \delta_{0}\right)$ there exists a transformation $u_{\delta}$ of $u_{\delta}^{*}$ such that $f_{\delta}=u_{\delta}(0, x)$ satisfies:

$$
f_{\delta}=\alpha_{\delta} G_{0}+\varphi_{\delta}, \quad \lim _{\delta \rightarrow 0^{+}} \frac{\alpha_{\delta}}{\delta}=1,
$$

with $\varphi_{\delta}$ satisfying the orthogonality properties (1.9) and

$$
\forall \delta \in\left(0, \delta_{0}\right), \quad\left\|\varphi_{\delta}\right\|_{L^{2}} \leq C \delta^{1+\frac{2}{N}} .
$$

By "transformation" we mean a symmetry of (1.1) which is a combination of transformations of the form (1.4) and (1.5).

Remark 3.4. We will later improve the estimates on $\varphi_{\delta}$ and $\alpha_{\delta}$ and obtain (see (3.22), (3.24)):

$$
\forall \delta \in\left(0, \delta_{0}\right), \quad\left\|\varphi_{\delta}\right\|_{L^{2}} \leq C \delta^{1+\frac{4}{N}} \text { and }\left|\alpha_{\delta}-\delta\right| \leq C \delta^{1+\frac{4}{N}}
$$

Proof. The proof is divided into three steps.

Step 1. Closeness to $G_{0}$. In this step we show that if $\delta$ is small enough, there exists a transformation $v_{\delta}$ of $u_{\delta}^{*}$ which satisfies the maximizer equations (2.1) and

$$
\lim _{\delta \rightarrow 0} \delta^{-1}\left\|g_{\delta}-\delta G_{0}\right\|_{L^{2}}=0, \quad \text { where } \quad g_{\delta}(x)=v_{\delta}(0, x)
$$

Arguing by contradiction, we see that it is sufficient to show that for any sequence $\delta_{n} \rightarrow 0$ there exists (after extraction of a subsequence) a sequence of solutions $\left\{v_{\delta_{n}}\right\}_{n}$ that are obtained as transformations of $u_{\delta_{n}}^{*}$ and satisfy (3.10). 
By Claim 2.10 and Lemma 2.9, there exists a constant $C>0$ such that

$$
\left.\left|\iint\right| e^{i \frac{t}{2} \Delta} f_{\delta_{n}}^{*}\right|^{2+\frac{4}{N}} d t d x-C_{S} \delta_{n}^{2+\frac{4}{N}} \mid \leq C \delta_{n}^{2+\frac{8}{N}} .
$$

By Lemma 3.2, we obtain after extraction of subsequences that there exist $\theta_{0} \in \mathbb{R}$ and a sequence of transformations $\left\{\Gamma_{n}\right\}$ such that

$$
\lim _{n \rightarrow \infty} \delta_{n}^{-1}\left\|f_{\delta_{n}}^{*}-\delta_{n} e^{i \theta_{0}} \Gamma_{n}(G)_{\mid t=0}\right\|_{L^{2}}=0 .
$$

Note that, by (1.6),

$$
\Gamma_{n}(G)_{\mid t=0}=\rho_{n}^{\frac{N}{2}} e^{i x \cdot \xi_{n}} G\left(t_{n}, \rho_{n} x+x_{n}\right)=\frac{\rho_{n}^{\frac{N}{2}} e^{i x \cdot \xi_{n}}}{\pi^{N / 4}\left(1+i t_{n}\right)^{N / 2}} e^{-\frac{\left|\rho_{n} x+x_{n}\right|^{2}}{2\left(1+i t_{n}\right)}} .
$$

And thus, by the change of variable $y=\frac{\rho_{n} x+x_{n}}{\sqrt{1+t_{n}^{2}}}$,

$$
\begin{aligned}
& \left\|f_{\delta_{n}}^{*}(x)-\delta_{n} e^{i \theta_{0}} \Gamma_{n}(G)_{\mid t=0}\right\|_{L^{2}}^{2} \\
& =\int\left|e^{i \tau_{n}+i \frac{t_{n}|y|^{2}}{2}+i \frac{\sqrt{1+t_{n}^{2}} y-x_{n}}{\rho_{n}} \cdot \xi_{n}} \frac{\left(1+t_{n}^{2}\right)^{\frac{N}{4}}}{\rho_{n}^{\frac{N}{2}}} \bar{f}_{\delta_{n}}^{*}\left(\frac{\sqrt{1+t_{n}^{2}} y-x_{n}}{\rho_{n}}\right)-\frac{\delta_{n} e^{-\frac{|y|^{2}}{2}}}{\pi^{\frac{N}{4}}}\right|^{2} d y,
\end{aligned}
$$

where $e^{i \tau_{n}}=\left(\frac{\sqrt{1+t_{n}^{2}}}{1+i t_{n}}\right)^{\frac{N}{2}}$. Consider the solution $w_{\delta_{n}}$ of (1.1) with initial condition

$$
h_{\delta_{n}}(x)=e^{i \tau_{n}+i \frac{\sqrt{1+t_{n}^{2}} y-x_{n}}{\rho_{n}}} \cdot \xi_{n} \frac{\left(1+t_{n}^{2}\right)^{\frac{N}{4}}}{\rho_{n}^{\frac{N}{2}}} \bar{f}_{\delta_{n}}^{*}\left(\frac{\sqrt{1+t_{n}^{2}} y-x_{n}}{\rho_{n}}\right),
$$

and the solution $v_{\delta_{n}}$ of (1.1) with initial condition $g_{\delta_{n}}=e^{i \frac{t_{n}|y|^{2}}{2}} h_{\delta_{n}}$. Then $w_{\delta_{n}}$ is an image of $u_{\delta_{n}}^{*}$ by phase, scaling, space translation and Galilean transformation (see (1.4)). Furthermore, $v_{\delta_{n}}$ is obtained from $w_{\delta_{n}}$ with a combination of pseudoconformal transformation and time translation. Namely:

$$
v_{\delta_{n}}(t, x)=\frac{t_{n}^{N / 2}}{\left(t_{n}^{2} t+t_{n}\right)^{N / 2}} e^{\frac{i t_{n}|x|^{2}}{2\left(t_{n} t+1\right)}} w_{\delta_{n}}\left(\frac{t}{1+t_{n} t}, \frac{t_{n} x}{t_{n}^{2} t+t_{n}}\right) .
$$

All these transformations preserve the $L^{2}$ norm and the global space-time $L^{2+\frac{4}{N}}$ norm, which shows that

$$
\left\|g_{\delta_{n}}\right\|_{L^{2}}=\delta_{n}, \quad \iint\left|v_{\delta_{n}}\right|^{\frac{4}{N}+2}=I\left(\delta_{n}\right) .
$$


By (3.11),

$$
\lim _{n \rightarrow \infty} \frac{1}{\delta_{n}}\left\|g_{\delta_{n}}-\delta_{n} G_{0}\right\|_{L^{2}}=0
$$

concluding the first step.

Step 2. Orthogonality conditions. We next show that the statement of the proposition holds if (3.9) is replaced by the weaker condition

$$
\lim _{\delta \rightarrow 0} \delta^{-1}\left\|\varphi_{\delta}\right\|_{L^{2}}=0 .
$$

For this we must show that there exists a transformation $u_{\delta}$ of $v_{\delta}$ such that $\varphi_{\delta}$ satisfies the orthogonality conditions (1.9). Consider the unit ball

$$
B_{L^{2}}\left(G_{0}, 1\right)=\left\{f \in L^{2},\left\|f-G_{0}\right\|_{L^{2}}<1\right\},
$$

and define, for small $\delta>0$, a differentiable mapping $\Phi_{\delta}: \mathbb{R} \times(0,+\infty) \times \mathbb{R} \times \mathbb{R}^{N} \times \mathbb{R}^{N} \times B_{L^{2}}\left(G_{0}, 1\right) \longrightarrow \mathbb{R} \times \mathbb{R} \times \mathbb{R}^{N} \times \mathbb{R}^{N} \times \mathbb{R}$ as follows. If $\theta_{0} \in \mathbb{R}, \Gamma_{0} \in(0,+\infty) \times \mathbb{R} \times \mathbb{R}^{N} \times \mathbb{R}^{N}, f \in B_{L^{2}}\left(G_{0}, 1\right), \tilde{u}_{\delta}$ is the solution of (1.1) with initial condition $\delta f$ and

$$
U_{\delta}(x)=\delta G_{0}-e^{i \theta_{0}} \Gamma_{0}\left(\tilde{u}_{\delta}\right)_{\mid t=0}=\delta G_{0}-e^{i \theta_{0}} \rho_{0}^{\frac{N}{2}} e^{i x \cdot \xi_{0}} \tilde{u}_{\delta}\left(t_{0}, \rho_{0} x+x_{0}\right),
$$

then $\Phi_{\delta}\left(\theta_{0}, \Gamma_{0}, f\right)=\left(\Phi_{\delta}^{1}, \Phi_{\delta}^{2}, \Phi_{\delta}^{3}, \Phi_{\delta}^{4}, \Phi_{\delta}^{5}\right)$ is defined by

$$
\begin{aligned}
& \Phi_{\delta}^{1}=\frac{1}{\delta} \operatorname{Im} \int U_{\delta} G_{0}, \quad \Phi_{\delta}^{2}=\frac{1}{\delta} \operatorname{Re} \int U_{\delta}\left(|x|^{2}-\frac{N}{2}\right) G_{0}, \quad \Phi_{\delta}^{3}=\frac{1}{\delta} \operatorname{Im} \int U_{\delta} x G_{0}, \\
& \Phi_{\delta}^{4}=\frac{1}{\delta} \operatorname{Re} \int U_{\delta} x G_{0}, \quad \Phi_{\delta}^{5}=\frac{1}{\delta} \operatorname{Im} \int U_{\delta}\left(|x|^{2}-\frac{N}{2}\right) G_{0} .
\end{aligned}
$$

Denote by $\Gamma_{i d}=(1,0,0,0)$ the identical transformation. Note that $\Phi_{\delta}\left(0, \Gamma_{i d}, G_{0}\right)=$ 0 . Then:

Claim 3.5. For small $\delta$, there exist $(\theta, \Gamma)$ close to $\left(0, \Gamma_{i d}\right)$ such that

$$
\Phi_{\delta}\left(\theta_{\delta}, \Gamma_{\delta}, \frac{1}{\delta} g_{\delta}\right)=0,
$$

where $g_{\delta}$ is the initial condition of the maximizer $v_{\delta}$ defined in Step 1.

We refer to Appendix A for the proof of Claim 3.5 which is based on a standard application of the implicit function theorem.

Let $u_{\delta}$ be the solution of (1.1) with initial condition

$$
f_{\delta}=e^{i \theta_{\delta}} \Gamma_{\delta}\left(v_{\delta}\right)_{\mid t=0} .
$$


Then by (3.10),

$$
\lim _{\delta \rightarrow \infty} \delta^{-1}\left\|f_{\delta}-\delta G_{0}\right\|_{L^{2}}=0
$$

Furthermore, from the invariance of the $L^{2}$ and $L_{t, x}^{2+\frac{4}{N}}$ norms by the transformations of the equation, $u_{\delta}$ satisfies the maximizer equations (2.1).

The fact that $\Phi_{\delta}\left(\theta_{\delta}, \Gamma_{\delta}, \delta^{-1} g_{\delta}\right)=0$ means that $f_{\delta}$ satisfies the orthogonality conditions

$$
\begin{gathered}
\operatorname{Im} \int\left(f_{\delta}-\delta G_{0}\right) G_{0}=0, \quad \int\left(f_{\delta}-\delta G_{0}\right) x G_{0}=0 \\
\int\left(f_{\delta}-\delta G_{0}\right)\left(|x|^{2}-\frac{N}{2}\right) G_{0}=0
\end{gathered}
$$

Let $\alpha_{\delta}=\operatorname{Re} \int f_{\delta} G_{0}$ and $\varphi_{\delta}=f_{\delta}-\alpha_{\delta} G_{0}$, so that $\operatorname{Re} \int \varphi_{\delta} G_{0}=0$. By (3.14) and (3.15), $\varphi_{\delta}$ satisfies the orthogonality conditions (1.9). By (3.13), $\lim _{\delta \rightarrow 0} \alpha_{\delta} / \delta=1$, which concludes Step 2.

Step 3. Proof of the estimate (3.9). In this step we conclude the proof of Proposition 3.3 using the coercivity of $Q$ (Theorem 1.5). To simplify notations, we will omit the index $\delta$ and write $u, f, \varphi$ and $\alpha$ instead of $u_{\delta}, f_{\delta}, \varphi_{\delta}$ and $\alpha_{\delta}$. All estimates stated hold for small $\delta>0$.

By Claim 2.10,

$$
\begin{aligned}
\left.\left|\iint\right| u\right|^{2+\frac{4}{N}} d t d x-\iint \mid e^{i \frac{t}{2} \Delta} f d t & \left.d x\right|^{2+\frac{4}{N}} \mid \\
& \leq C \delta^{1+\frac{4}{N}}\left\|u-e^{i \frac{t}{2} \Delta} f\right\|_{L_{t, x}^{2+\frac{4}{N}}} \leq C \delta^{2+\frac{8}{N}} .
\end{aligned}
$$

Recalling that $\frac{1}{\alpha} f=G_{0}(x)+\frac{1}{\alpha} \varphi$ and using the expansion of the Strichartz norm, we obtain

$$
\begin{aligned}
\iint|u|^{2+\frac{4}{N}} d t d x= & \alpha^{2+\frac{4}{N}} \iint\left|e^{i \frac{t}{2} \Delta} \frac{1}{\alpha} f\right|^{2+\frac{4}{N}} d t d x+\mathcal{O}\left(\delta^{2+\frac{8}{N}}\right) \\
= & C_{S}\left(\int|f|^{2} d x\right)^{1+\frac{2}{N}}-\alpha^{2+\frac{4}{N}} Q\left(\frac{1}{\alpha} \varphi\right) \\
& +\alpha^{2+\frac{4}{N}} \mathcal{O}\left(\frac{1}{\alpha^{3}}\|\varphi\|_{L^{2}}^{3}\right)+\mathcal{O}\left(\delta^{2+\frac{8}{N}}\right) \\
= & C_{S} \delta^{2+\frac{4}{N}}-\alpha^{\frac{4}{N}} Q(\varphi)+\mathcal{O}\left(\alpha^{\frac{4}{N}-1}\|\varphi\|_{L^{2}}^{3}\right)+\mathcal{O}\left(\delta^{2+\frac{8}{N}}\right) .
\end{aligned}
$$

Using that $u$ satisfies (2.1), we get

$$
\iint|u|^{2+\frac{4}{N}} d t d x=I(\delta)=C_{S} \delta^{2+\frac{4}{N}}+\mathcal{O}\left(\delta^{2+\frac{8}{N}}\right),
$$


and thus,

$\alpha^{\frac{4}{N}} Q(\varphi)=\mathcal{O}\left(\alpha^{\frac{4}{N}-1}\|\varphi\|_{L^{2}}^{3}\right)+\mathcal{O}\left(\delta^{2+\frac{8}{N}}\right)=\mathcal{O}\left(\alpha^{\frac{4}{N}}\|\varphi\|_{L^{2}}^{2} \frac{\|\varphi\|_{L^{2}}}{\alpha}\right)+\mathcal{O}\left(\delta^{2+\frac{8}{N}}\right)$.

By Theorem 1.5, $\|\varphi\|_{L^{2}}^{2} \lesssim Q(\varphi)$, and thus, using that $\frac{1}{\alpha}\|\varphi\|_{L^{2}} \rightarrow 0$ as $\delta \rightarrow 0$,

$$
\alpha^{\frac{4}{N}}\|\varphi\|_{L^{2}}^{2}=\mathcal{O}\left(\delta^{2+\frac{8}{N}}\right)
$$

which shows (3.9).

\subsection{Proof of the estimate on the maximum}

The idea of the proof of Proposition 3.1 is to compare $I(\delta)$ with the $L^{2+\frac{4}{N}}$ norm of $H_{\delta}$, the solution to the nonlinear equation (1.1) with the Gaussian initial data $\delta G_{0}$. We have

$$
\iint\left|u_{\delta}\right|^{2+\frac{4}{N}} d t d x=I(\delta) \geq \iint\left|H_{\delta}\right|^{2+\frac{4}{N}} d t d x .
$$

The global $L^{2+\frac{4}{N}}$ of $H_{\delta}$ may be estimated as follows:

Lemma 3.6. Let

$$
D_{N}=-\left(2+\frac{4}{N}\right) \operatorname{Im} \iint|G(t)|^{\frac{4}{N}} \bar{G}(t) \int_{0}^{t} e^{i \frac{(t-s)}{2} \Delta}\left(|G(s)|^{\frac{4}{N}} G(s)\right) d s d t d x .
$$

Then for small $\delta>0$,

$$
\iint\left|H_{\delta}\right|^{2+\frac{4}{N}} d t d x=\delta^{2+\frac{4}{N}} \iint|G|^{2+\frac{4}{N}} d t d x+\gamma D_{N} \delta^{2+\frac{8}{N}}+\mathcal{O}\left(\delta^{2+\frac{12}{N}}\right) .
$$

The exact value of the constant $D_{N}$ will be computed in Appendix B (dimension 1) and Appendix C (dimension 2).

Proof of Lemma 3.6. Since $G$ is the linear evolution of $G_{0}$, we have

$$
H_{\delta}=\delta G+i \gamma \int_{0}^{t} e^{i \frac{(t-s)}{2} \Delta}\left|H_{\delta}(s)\right|^{\frac{4}{N}} H_{\delta}(s) d s .
$$

We approximate $H_{\delta}$ by $v_{\delta}$ :

$$
v_{\delta}(t, x)=\delta\left(G(t, x)+\gamma \delta^{\frac{4}{N}} r(t, x)\right),
$$

where

$$
r(t, x)=i \int_{0}^{t} e^{i \frac{(t-s)}{2} \Delta}|G(s)|^{\frac{4}{N}} G(s) d s,
$$


in other words, $v_{\delta}$ solves

$$
i \partial_{t} v_{\delta}+\frac{1}{2} \Delta v_{\delta}+\gamma \delta^{\frac{4}{N}+1}|G|^{\frac{4}{N}} G=0, \quad v_{\delta}(0, x)=\delta G_{0}(x)
$$

and $r$ solves

$$
i \partial_{t} r+\frac{1}{2} \Delta r+|G|^{\frac{4}{N}} G=0, \quad r(0, x)=0 .
$$

Since by Claim 2.10

$$
\begin{aligned}
& \left\|\left|H_{\delta}\right|^{\frac{4}{N}} H_{\delta}-\delta^{\frac{4}{N}+1}|G|^{\frac{4}{N}} G\right\| \|_{L_{t, x}^{\frac{2(N+2)}{N+4}}} \\
& \leq C\left\|H_{\delta}-\delta G\right\| \underset{L_{t, x}^{2+\frac{4}{N}}}{ }\left(\left\|H_{\delta}\right\|_{L_{t, x}^{2+\frac{4}{N}}}^{\frac{4}{N}}+\|\delta G\|_{L_{t, x}^{2+\frac{4}{N}}}^{\frac{4}{N}}\right) \leq C \delta^{\frac{8}{N}+1},
\end{aligned}
$$

by Strichartz estimates, we have

$$
\left\|H_{\delta}-v_{\delta}\right\|_{L_{t, x}^{2+\frac{4}{N}}} \leq C \delta^{1+\frac{8}{N}}
$$

and thus,

$$
\left.\left|\iint\right| H_{\delta}\right|^{2+\frac{4}{N}} d t d x-\iint\left|v_{\delta}\right|^{2+\frac{4}{N}} d t d x \mid \lesssim\left\|H_{\delta}-v_{\delta}\right\|_{L_{t, x}^{2+\frac{4}{N}}}\left\|\delta G_{0}\right\|_{L^{2}}^{1+\frac{4}{N}} \lesssim \delta^{2+\frac{12}{N}}
$$

which is exactly the power of higher order terms in (3.17). It remains to estimate $\iint\left|v_{\delta}\right|^{2+\frac{4}{N}}$. Note that if $A$ and $B$ are functions of space and time,

$$
\begin{aligned}
& \iint|A+B|^{2+\frac{4}{N}} \\
& \quad=\iint|A|^{2+\frac{4}{N}}+\left(2+\frac{4}{N}\right) \operatorname{Re} \iint|A|^{\frac{4}{N}} A \bar{B}+\mathcal{O}\left(\iint|A|^{\frac{4}{N}}|B|^{2}+|B|^{2+\frac{4}{N}}\right) .
\end{aligned}
$$

By (3.19) and the definition of $v_{\delta}$ we get,

$$
\begin{aligned}
\iint\left|v_{\delta}\right|^{2+\frac{4}{N}} d t d x= & \delta^{2+\frac{4}{N}} \iint|G|^{2+\frac{4}{N}} d t d x \\
& +\delta^{2+\frac{8}{N}}\left(2+\frac{4}{N}\right) \operatorname{Re} \iint|G|^{\frac{4}{N}} \bar{G} r d t d x+\mathcal{O}\left(\delta^{2+\frac{12}{N}}\right),
\end{aligned}
$$

which concludes the proof of Lemma 3.6 in view of the definition (3.16) of $D_{N}$. 
We next prove Proposition 3.1. Let $u_{\delta}, f_{\delta}, \varphi_{\delta}$ and $\alpha_{\delta}$ be as in Proposition 3.3. We have

$$
u_{\delta}=\underbrace{e^{i \frac{t}{2} \Delta}\left(\alpha_{\delta} G_{0}+\varphi_{\delta}\right)}_{A}+\underbrace{i \gamma \int_{0}^{t} e^{i \frac{(t-s)}{2} \Delta}\left(\left|u_{\delta}(s)\right|^{\frac{4}{N}} u_{\delta}(s)\right) d s}_{B}
$$

By (3.9) and Strichartz estimate (1.3),

$$
\left\|e^{i \frac{t}{2} \Delta} \varphi_{\delta}\right\|_{L^{2+\frac{4}{N}}} \leq C\left\|\varphi_{\delta}\right\|_{L^{2}} \leq C \delta^{1+\frac{2}{N}}
$$

Expanding the $B$ term in (3.20) and applying Strichartz estimates again to bound the terms in $\varphi_{\delta}$, we get (the $\mathcal{O}$ 's are estimated in the space $L_{t, x}^{2+\frac{4}{N}}$ ).

$$
\begin{aligned}
B & =i \gamma \int_{0}^{t} e^{i \frac{(t-s)}{2} \Delta}\left(\left|u_{\delta}(s)\right|^{\frac{4}{N}} u_{\delta}(s)\right) d s \\
& =i \gamma \int_{0}^{t} e^{i \frac{(t-s)}{2} \Delta}\left[\left|\alpha_{\delta} G(s)+e^{i \frac{s}{2} \Delta} \varphi_{\delta}\right|^{\frac{4}{N}}\left(\alpha_{\delta} G(s)+e^{i \frac{s}{2} \Delta} \varphi_{\delta}\right)\right] d s+\mathcal{O}\left(\delta^{1+\frac{8}{N}}\right) \\
& =i \gamma \alpha_{\delta}^{1+\frac{4}{N}} \int_{0}^{t} e^{i \frac{(t-s)}{2} \Delta}\left(|G(s)|^{\frac{4}{N}} G(s)\right) d s+\mathcal{O}\left(\delta^{\frac{4}{N}}\left\|\varphi_{\delta}\right\|_{L^{2}}+\delta^{1+\frac{8}{N}}\right) .
\end{aligned}
$$

And thus, by (3.19) and (3.20),

$$
\begin{aligned}
& \iint\left|u_{\delta}\right|^{2+\frac{4}{N}} d t d x=\iint\left|\alpha_{\delta} G+e^{i \frac{t}{2} \Delta} \varphi_{\delta}\right|^{2+\frac{4}{N}} d t d x \\
& -\left(2+\frac{4}{N}\right) \gamma \alpha_{\delta}^{2+\frac{8}{N}} \operatorname{Im} \iint|G(t)|^{\frac{4}{N}} \bar{G}(t) \int_{0}^{t} e^{i \frac{(t-s)}{2} \Delta}\left(|G(s)|^{\frac{4}{N}} G(s)\right) d s d t d x \\
& +\mathcal{O}\left(\delta^{1+\frac{8}{N}}\left\|\varphi_{\delta}\right\|_{L^{2}}\right)+\mathcal{O}\left(\delta^{2+\frac{12}{N}}\right) .
\end{aligned}
$$

By the equation (3.2)

$$
\begin{aligned}
\iint \mid \alpha_{\delta} G & +\left.e^{i \frac{t}{2} \Delta} \varphi_{\delta}\right|^{2+\frac{4}{N}} d t d x \\
& =\alpha_{\delta}^{2+\frac{4}{N}}\left[C_{S}\left\|G_{0}+\frac{1}{\alpha_{\delta}} \varphi_{\delta}\right\|_{L^{2}}^{2+\frac{4}{N}}-Q\left(\frac{1}{\alpha_{\delta}} \varphi_{\delta}\right)+\mathcal{O}\left(\frac{1}{\alpha_{\delta}^{3}}\left\|\varphi_{\delta}\right\|_{L^{2}}^{3}\right)\right] .
\end{aligned}
$$

By (3.9) and (3.21), using that

$$
\left\|\alpha_{\delta} G_{0}+\varphi_{\delta}\right\|_{L^{2}}^{2}=\delta^{2}=\alpha_{\delta}^{2}+\left\|\varphi_{\delta}\right\|_{L^{2}}^{2}=\alpha_{\delta}^{2}+\mathcal{O}\left(\delta^{2+\frac{4}{N}}\right)
$$


we get, in view of the definition (3.16) of $D_{N}$,

$$
\begin{aligned}
& \iint\left|u_{\delta}\right|^{2+\frac{4}{N}} d t d x \\
& =C_{S} \delta^{2+\frac{4}{N}}-\alpha_{\delta}^{\frac{4}{N}} Q\left(\varphi_{\delta}\right)+\gamma D_{N} \alpha_{\delta}^{2+\frac{8}{N}}+\mathcal{O}\left(\delta^{1+\frac{8}{N}}\left\|\varphi_{\delta}\right\|_{L^{2}}\right)+\mathcal{O}\left(\delta^{2+\frac{12}{N}}\right) .
\end{aligned}
$$

By Lemma 3.6,

$$
\iint\left|u_{\delta}\right|^{2+\frac{4}{N}} d t d x \geq C_{S} \delta^{2+\frac{4}{N}}+\gamma D_{N} \delta^{2+\frac{8}{N}}+\mathcal{O}\left(\delta^{2+\frac{12}{N}}\right)
$$

Combining with (3.23), we get

$$
\begin{aligned}
C_{S} \delta^{2+\frac{4}{N}}-\alpha_{\delta}^{\frac{4}{N}} Q\left(\varphi_{\delta}\right) & +\gamma D_{N} \alpha_{\delta}^{2+\frac{8}{N}}+\mathcal{O}\left(\delta^{2+\frac{12}{N}}\right)+\mathcal{O}\left(\delta^{1+\frac{8}{N}}\left\|\varphi_{\delta}\right\|_{L^{2}}\right) \\
& \geq C_{S} \delta^{2+\frac{4}{N}}+\gamma D_{N} \delta^{2+\frac{8}{N}}
\end{aligned}
$$

Using that by (3.22)

$$
\left|\delta^{2+\frac{8}{N}}-\alpha_{\delta}^{2+\frac{8}{N}}\right|=\mathcal{O}\left(\delta^{2+\frac{12}{N}}\right)
$$

this simplifies to

$$
\alpha_{\delta}^{\frac{4}{N}} Q\left(\varphi_{\delta}\right)=\mathcal{O}\left(\delta^{2+\frac{12}{N}}\right)+\mathcal{O}\left(\delta^{1+\frac{8}{N}}\left\|\varphi_{\delta}\right\|_{L^{2}}\right)
$$

Let $X=\left\|\varphi_{\delta}\right\|_{L^{2}} \delta^{-1-\frac{4}{N}}$. By the preceding estimate and Theorem 1.5, there exists a constant $C>0$ independent of $\delta$ such that $X^{2} \leq C(1+X)$. This implies that $X$ is bounded independently of $\delta$, i.e.

$$
\left\|\varphi_{\delta}\right\|_{L^{2}}=\mathcal{O}\left(\delta^{1+\frac{4}{N}}\right)
$$

By (3.23) again,

$$
I(\delta)=\iint\left|u_{\delta}\right|^{2+\frac{4}{N}}=C_{S} \delta^{2+\frac{4}{N}}+\gamma D_{N} \delta^{2+\frac{8}{N}}+\mathcal{O}\left(\delta^{2+\frac{12}{N}}\right) .
$$

The proof is complete, except for the computation of $D_{N}$ which is given in appendices B and C. Note that as announced in Remark 3.4, the estimate (3.24) improves the preceding estimate (3.9) on $\varphi_{\delta}$. 


\section{Uniqueness}

In this section we show the uniqueness part of Theorem 1.1. We assume again

$$
N \in\{1,2\} .
$$

By Proposition 2.1, there exists, for small $\delta>0$, a maximizer for $I(\delta)$, i.e. a solution $u_{\delta}$ of (1.1) such that

$$
\left\|f_{\delta}\right\|_{L^{2}}=\delta, \quad \iint\left|u_{\delta}\right|^{2+\frac{4}{N}}=I(\delta)
$$

(as usual $f_{\delta}(x)=u_{\delta}(0, x)$ ). By Proposition 3.3 and Remark 3.4, assuming again that $\delta$ is small, any maximizer for $I(\delta)$ satisfies, after transformation, the following properties:

$$
f_{\delta}=\alpha_{\delta} G_{0}+\varphi_{\delta}
$$

where $\varphi_{\delta} \in L^{2}\left(\mathbb{R}^{N}\right)$ and $\alpha_{\delta}>0$ are such that

$$
\begin{gathered}
\int \varphi G_{0}=\int \varphi|x|^{2} G_{0}=0, \quad \int \varphi x G_{0}=0_{\mathbb{R}^{N}}, \\
\left\|\varphi_{\delta}\right\|_{L^{2}} \leq C \delta^{1+\frac{4}{N}}, \quad \alpha_{\delta}>0 \quad \text { and } \quad\left|\alpha_{\delta}-\delta\right| \leq C \delta^{1+\frac{4}{N}} .
\end{gathered}
$$

We must show that if $C>0$, there exists $\delta_{0}>0$ such that if $\delta \in\left(0, \delta_{0}\right)$, there is at most one solution $u_{\delta}$ of (1.1) satisfying (4.1), (4.2), (4.3) and (4.4).

Let us fix a small $\delta>0$ and a maximizer $u_{\delta}$ satisfying (4.1), (4.2), (4.3) and (4.4). The strategy of the proof is to expand $\int|v|^{2+\frac{4}{n}}$, where $v$ is a solution of (1.1) which is close to $u_{\delta}$. In Section 4.1 we expand $v$ and $\int|v|^{2+\frac{4}{n}}$ at first order, in Section 4.2 we obtain a second order expansion involving the quadratic form $Q$. Assuming that $v$ is another maximizer, the conclusion will follow from Theorem 1.5.

\subsection{Linearization}

Lemma 4.1. There exists a linear operator $L_{\delta}: L_{t, x}^{2+\frac{4}{N}} \rightarrow L_{t, x}^{2+\frac{4}{N}}$ such that

$$
\forall h \in L_{t, x}^{2+\frac{4}{N}}, \quad\left\|\left(L_{\delta}-1\right) h\right\|_{L_{t, x}^{2+\frac{4}{N}}} \leq C \delta^{\frac{4}{N}}\|h\|_{L_{t, x}^{2+\frac{4}{N}}},
$$

with the following property: if $v$ is a solution of (1.1) with the initial condition $f_{\delta}+\psi$, where

$$
\|\psi\|_{L^{2}} \leq \delta
$$

then

$$
\left\|v-u_{\delta}-L_{\delta}\left(e^{i \frac{t}{2} \Delta} \psi\right)\right\|_{L_{t, x}^{2+\frac{4}{N}}} \leq C \delta^{\frac{4}{N}-1}\|\psi\|_{L^{2}}^{2} .
$$


Proof. Let $w=v-u_{\delta}$. Then by Lemma 2.2,

$$
\|w\|_{L_{t, x}^{2+\frac{4}{N}}} \leq C\|\psi\|_{L^{2}}
$$

Writing Duhamel's formula for $u_{\delta}$ and $v=u_{\delta}+w$, we get $w=e^{i \frac{t}{2} \Delta} \psi+i \gamma \int_{0}^{t} e^{i \frac{(t-s)}{2} \Delta}\left(\left|u_{\delta}(s)+w(s)\right|^{\frac{4}{N}}\left(u_{\delta}(s)+w(s)\right)-\left|u_{\delta}(s)\right|^{\frac{4}{N}} u_{\delta}(s)\right) d s$. Expanding $\left|u_{\delta}(s)+w(s)\right|^{\frac{4}{N}}\left(u_{\delta}(s)+w(s)\right)$, one can write the preceding equality as

$$
w=e^{i \frac{t}{2} \Delta} \psi+\widetilde{L_{\delta}} w+\widetilde{R_{\delta}}(w)
$$

where the linear operator $\widetilde{L_{\delta}}: L_{t, x}^{2+\frac{4}{N}} \rightarrow L_{t, x}^{2+\frac{4}{N}}$ satisfies

$$
\left\|\widetilde{L_{\delta}} w\right\|_{L_{t, x}^{2+\frac{4}{N}}} \leq C \delta^{\frac{4}{N}}\|w\|_{L_{t, x}^{2+\frac{4}{N}}}
$$

and $\widetilde{R_{\delta}}$ satisfies

$$
\left\|\widetilde{R_{\delta}}(w)\right\| \leq C\left(\begin{array}{r}
\delta^{\frac{4}{N}-1}\|w\|_{L_{t, x}^{2+\frac{4}{N}}}^{2}+\|w\|_{L_{t, x}^{2+\frac{4}{N}}}^{1+\frac{4}{N}} \\
{ }^{2+2}
\end{array}\right)
$$

Letting for small $\delta$

$$
L_{\delta}=\left(1-\widetilde{L_{\delta}}\right)^{-1}
$$

we obtain by (4.10) that $L_{\delta}$ satisfies (4.5). The estimate (4.7) follows from (4.6), (4.8), (4.9) and (4.11).

Lemma 4.2. Let $L_{\delta}$ be as in Lemma 4.1. Then for small $\delta>0$,

$$
\operatorname{Re} \iint\left|u_{\delta}\right|^{\frac{4}{N}} \overline{u_{\delta}} L_{\delta}\left(e^{i \frac{t}{2} \Delta} \psi\right)=\mu_{\delta} \operatorname{Re} \int \overline{f_{\delta}} \psi,
$$

where $\mu_{\delta}>0$, which depends only on $u_{\delta}$, satisfies

$$
\left|\mu_{\delta}-C_{S} \delta^{\frac{4}{N}}\right| \leq C \delta^{\frac{8}{N}}
$$

Proof. Indeed, by definition

$$
I(\delta)=\max \iint|v|^{2+\frac{4}{N}},
$$


where the maximum is taken over all solutions $v$ of (1.1) with initial condition $f_{\delta}+\psi$, such that $\int\left|f_{\delta}+\psi\right|^{2}=\delta^{2}$. For such a solution $v$, write, as in the proof of Lemma 4.1, $v=u_{\delta}+w$. Then

$$
\begin{aligned}
\iint|v|^{2+\frac{4}{N}}= & \iint\left|u_{\delta}+w\right|^{2+\frac{4}{N}} \\
= & \iint\left|u_{\delta}\right|^{2+\frac{4}{N}}+\left(2+\frac{4}{N}\right) \operatorname{Re} \iint\left|u_{\delta}\right|^{\frac{4}{N}} \overline{u_{\delta}} w+\mathcal{O}\left(\delta^{\frac{4}{N}}\|\psi\|_{L^{2}}^{2}\right) \\
= & \iint\left|u_{\delta}\right|^{2+\frac{4}{N}}+\left(2+\frac{4}{N}\right) \operatorname{Re} \iint\left|u_{\delta}\right|^{\frac{4}{N}} \overline{u_{\delta}} L_{\delta}\left(e^{i \frac{t}{2} \Delta} \psi\right) \\
& +\mathcal{O}\left(\delta^{\frac{4}{N}}\|\psi\|_{L^{2}}^{2}\right) .
\end{aligned}
$$

The existence of $\mu_{\delta}$ then follows from the Lagrange multiplier equation for the maximizing problem (4.14).

We next estimate $\mu_{\delta}$. By (4.2) and (4.4)

$$
f_{\delta}=\delta G_{0}+\mathcal{O}\left(\delta^{1+\frac{4}{N}}\right) \text { in } L^{2} .
$$

Thus by Claim 2.10,

$$
u_{\delta}=\delta G+\mathcal{O}\left(\delta^{1+\frac{4}{N}}\right) \text { in } L_{t, x}^{2+\frac{4}{N}} .
$$

As a consequence, we obtain (assuming $\|\psi\|_{L^{2}} \leq \delta$ )

$$
\begin{aligned}
\operatorname{Re} \iint\left|u_{\delta}\right|^{\frac{4}{N}} \bar{u}_{\delta} L_{\delta}\left(e^{i \frac{t}{2} \Delta} \psi\right) & =\operatorname{Re} \iint\left|u_{\delta}\right|^{\frac{4}{N}} \bar{u}_{\delta} e^{i \frac{t}{2} \Delta} \psi+\mathcal{O}\left(\delta^{1+\frac{8}{N}}\|\psi\|_{L^{2}}\right) \\
& =\delta^{1+\frac{4}{N}} \operatorname{Re} \iint|G|^{\frac{4}{N}} \bar{G} e^{i \frac{t}{2} \Delta} \psi+\mathcal{O}\left(\delta^{1+\frac{8}{N}}\|\psi\|_{L^{2}}\right) .
\end{aligned}
$$

On the other hand,

$\operatorname{Re} \int \bar{f}_{\delta} \psi=\operatorname{Re} \int \alpha_{\delta} G_{0} \psi+\mathcal{O}\left(\delta^{1+\frac{4}{N}}\|\psi\|_{L^{2}}\right)=\delta \operatorname{Re} \int G_{0} \psi+\mathcal{O}\left(\delta^{1+\frac{4}{N}}\|\psi\|_{L^{2}}\right)$.

Combining with (4.12), we get

$\delta^{1+\frac{4}{N}} \operatorname{Re} \iint|G|^{\frac{4}{N}} \bar{G} e^{i \frac{t}{2} \Delta} \psi=\delta \mu_{\delta} \operatorname{Re} \int G_{0} \psi+\mathcal{O}\left(\delta^{1+\frac{8}{N}}\|\psi\|_{L^{2}}+\mu_{\delta} \delta^{1+\frac{4}{N}}\|\psi\|_{L^{2}}\right)$.

By (3.1),

$$
C_{S} \delta^{1+\frac{4}{N}} \operatorname{Re} \int G_{0} \psi=\delta \mu_{\delta} \operatorname{Re} \int G_{0} \psi+\mathcal{O}\left(\mu_{\delta} \delta^{1+\frac{4}{N}}\|\psi\|_{L^{2}}+\delta^{1+\frac{8}{N}}\|\psi\|_{L^{2}}\right) .
$$

This holds for all small $\psi \in L^{2}$, yielding (4.13). 


\subsection{Second order expansion}

Lemma 4.3. Let $v$ be a solution of (1.1) with initial condition $f_{\delta}+\psi$, and assume

$$
\int\left|f_{\delta}+\psi\right|^{2}=\delta^{2}
$$

Then

$$
\iint|v|^{2+\frac{4}{N}}=I(\delta)-\delta^{\frac{4}{N}} Q(\psi)+\mathcal{O}\left(\delta^{\frac{8}{N}}\|\psi\|_{L^{2}}^{2}+\delta^{\frac{4}{N}-1}\|\psi\|_{L^{2}}^{3}+\|\psi\|_{L^{2}}^{2+\frac{4}{N}}\right) .
$$

Proof. Using that $\int\left|f_{\delta}\right|^{2}=\delta^{2}$, we get

$$
\int|\psi|^{2}=-2 \operatorname{Re} \int \overline{f_{\delta}} \psi
$$

and thus by (4.2) and (4.4),

$$
\delta^{2}\left|\operatorname{Re} \int G_{0} \psi\right|^{2} \leq C\left(\delta^{\frac{8}{N}+2}\|\psi\|_{L^{2}}^{2}+\|\psi\|_{L^{2}}^{4}\right) .
$$

Expanding $\left|u_{\delta}+w\right|^{2+\frac{4}{N}}$ at second order in $w$, we obtain

$$
\begin{aligned}
\iint|v|^{2+\frac{4}{N}}= & \iint\left|u_{\delta}+w\right|^{2+\frac{4}{N}} \\
= & \iint\left|u_{\delta}\right|^{2+\frac{4}{N}}+\left(2+\frac{4}{N}\right) \operatorname{Re} \iint\left|u_{\delta}\right|^{\frac{4}{N}} \bar{u}_{\delta} w \\
& +\left(1+\frac{2}{N}\right)^{2} \iint\left|u_{\delta}\right|^{\frac{4}{N}}|w|^{2}+\frac{2}{N}\left(1+\frac{2}{N}\right) \operatorname{Re} \iint\left|u_{\delta}\right|^{\frac{4}{N}-2} \bar{u}_{\delta}^{2} w^{2} \\
& +\mathcal{O}\left(\delta^{\frac{4}{N}-1}\|\psi\|_{L^{2}}^{3}\right)+\mathcal{O}\left(\|\psi\|_{L^{2}}^{2+\frac{4}{N}}\right) .
\end{aligned}
$$

By Lemma 4.1, Lemma 4.2 and (4.17),

$$
\begin{aligned}
\operatorname{Re} \iint\left|u_{\delta}\right|^{\frac{4}{N}} \bar{u}_{\delta} w & =\operatorname{Re} \iint\left|u_{\delta}\right|^{\frac{4}{N}} \bar{u}_{\delta} L_{\delta}\left(e^{i \frac{t}{2} \Delta} \psi\right)+\mathcal{O}\left(\delta^{\frac{8}{N}}\|\psi\|_{L^{2}}^{2}\right) \\
& =\mu_{\delta} \int \bar{f}_{\delta} \psi+\mathcal{O}\left(\delta^{\frac{8}{N}}\|\psi\|_{L^{2}}^{2}\right) \\
& =-\frac{\mu_{\delta}}{2} \int|\psi|^{2}+\mathcal{O}\left(\delta^{\frac{8}{N}}\|\psi\|_{L^{2}}^{2}\right) \\
& =-\frac{C_{S}}{2} \delta^{\frac{4}{N}} \int|\psi|^{2}+\mathcal{O}\left(\delta^{\frac{8}{N}}\|\psi\|_{L^{2}}^{2}\right) .
\end{aligned}
$$


By (4.15), then Lemma 4.1,

$$
\begin{aligned}
\iint\left|u_{\delta}\right|^{\frac{4}{N}}|w|^{2} & =\delta^{\frac{4}{N}} \iint|G|^{\frac{4}{N}}|w|^{2}+\mathcal{O}\left(\delta^{\frac{8}{N}}\|\psi\|_{L^{2}}^{2}\right) \\
& =\delta^{\frac{4}{N}} \iint|G|^{\frac{4}{N}}\left|e^{i \frac{t}{2} \Delta} \psi\right|^{2}+\mathcal{O}\left(\delta^{\frac{8}{N}}\|\psi\|_{L^{2}}^{2}\right),
\end{aligned}
$$

and similarly

$$
\operatorname{Re} \iint\left|u_{\delta}\right|^{\frac{4}{N}-2} \bar{u}_{\delta}^{2} w^{2}=\delta^{\frac{4}{N}} \operatorname{Re} \iint \mid G^{\frac{4}{N}-2} \bar{G}^{2}\left(e^{i \frac{t}{2} \Delta} \psi\right)^{2}+\mathcal{O}\left(\delta^{\frac{8}{N}}\|\psi\|_{L^{2}}^{2}\right) .
$$

Combining the preceding estimates, we obtain

$$
\begin{aligned}
\iint|v|^{2+\frac{4}{N}}= & \iint\left|u_{\delta}\right|^{2+\frac{4}{N}}-C_{S}\left(\frac{N+2}{N}\right) \delta^{\frac{4}{N}} \int|\psi|^{2} \\
& +\left(1+\frac{2}{N}\right)^{2} \delta^{\frac{4}{N}} \iint|G|^{\frac{4}{N}}\left|e^{i \frac{t}{2} \Delta} \psi\right|^{2} \\
& +\frac{2}{N}\left(1+\frac{2}{N}\right) \delta^{\frac{4}{N}} \operatorname{Re} \iint|G|^{\frac{4}{N}-2} \bar{G}^{2}\left(e^{i \frac{t}{2} \Delta} \psi\right)^{2} \\
& +\mathcal{O}\left(\delta \frac{8}{N}\|\psi\|_{L^{2}}^{2}+\delta \frac{4}{N}-1\|\psi\|_{L^{2}}^{3}+\|\psi\|_{L^{2}}^{2+\frac{4}{N}}\right),
\end{aligned}
$$

which yields (4.16) in view of (4.18) and the definition (3.3) of $Q$.

We can now conclude the proof of the uniqueness of the maximizer. Assume that $\delta>0$ is small and consider a solution $\tilde{u}_{\delta}$ of (1.1) with initial condition $\tilde{f}_{\delta}=$ $\tilde{\alpha}_{\delta} G_{0}+\tilde{\varphi}_{\delta}$. Assume that $\tilde{u}_{\delta}, \tilde{f}_{\delta}, \tilde{\varphi}_{\delta}$ and $\tilde{\alpha}_{\delta}$ also satisfy (4.1), (4.2), (4.3) and (4.4). We must show that $\tilde{u}_{\delta}=u_{\delta}$. Let

$$
\psi=\left(\tilde{\alpha}_{\delta}-\alpha_{\delta}\right) G_{0}+\tilde{\varphi}_{\delta}-\varphi_{\delta}
$$

By (4.4), $\|\psi\|_{L^{2}} \leq C \delta^{\frac{4}{N}+1}$. By Lemma 4.3 with $v=\tilde{u}_{\delta}$,

$$
I(\delta)=\iint|v|^{2+\frac{4}{N}}=I(\delta)-\delta^{\frac{4}{N}} Q(\psi)+\mathcal{O}\left(\delta \frac{8}{N}\|\psi\|_{L^{2}}^{2}+\delta^{\frac{4}{N}-1}\|\psi\|_{L^{2}}^{3}+\|\psi\|_{L^{2}}^{2+\frac{4}{N}}\right),
$$

and thus,

$$
\delta^{\frac{4}{N}} Q(\psi) \leq C\left(\delta^{\frac{8}{N}}\|\psi\|_{L^{2}}^{2}+\delta^{\frac{4}{N}-1}\|\psi\|_{L^{2}}^{3}+\|\psi\|_{L^{2}}^{2+\frac{4}{N}}\right) \leq C \delta^{\frac{8}{N}}\|\psi\|_{L^{2}}^{2} .
$$

Since $G_{0}$ is in the kernel of $Q, Q(\psi)=Q\left(\tilde{\varphi}_{\delta}-\varphi_{\delta}\right)$. Using that $\varphi_{\delta}$ and $\tilde{\varphi}_{\delta}$ satisfy the orthogonality conditions (1.9), we deduce from Theorem 1.5:

$$
c\left\|\tilde{\varphi}_{\delta}-\varphi_{\delta}\right\|^{2} \leq Q(\psi) .
$$


Using that

$$
\alpha_{\delta}^{2}+\int\left|\varphi_{\delta}\right|^{2}=\delta^{2}=\tilde{\alpha}_{\delta}^{2}+\int\left|\tilde{\varphi}_{\delta}\right|^{2},
$$

we obtain, in view of (4.4),

$$
\left|\tilde{\alpha}_{\delta}-\alpha_{\delta}\right|=\left|\frac{\tilde{\alpha}_{\delta}^{2}-\alpha_{\delta}^{2}}{\alpha_{\delta}+\tilde{\alpha}_{\delta}}\right| \leq\left.\frac{1}{\delta}\left|\int\right| \varphi_{\delta}\right|^{2}-\int\left|\tilde{\varphi}_{\delta}\right|^{2} \mid \leq C \delta^{\frac{4}{N}}\left\|\varphi_{\delta}-\tilde{\varphi}_{\delta}\right\|_{L^{2}}
$$

and thus for small $\delta$,

$$
\|\psi\|_{L^{2}}^{2}=\left(\tilde{\alpha}_{\delta}-\alpha_{\delta}\right)^{2}+\left\|\varphi_{\delta}-\tilde{\varphi}_{\delta}\right\|_{L^{2}}^{2} \leq 2\left\|\varphi_{\delta}-\tilde{\varphi}_{\delta}\right\|_{L^{2}}^{2} .
$$

Combining (4.19), (4.20) and (4.21), we get

$$
\delta^{\frac{4}{N}}\|\psi\|_{L^{2}}^{2} \leq C \delta^{\frac{8}{N}}\|\psi\|_{L^{2}}^{2}
$$

a contradiction if $\delta>0$ is small and $\psi \neq 0$. Thus, $\psi=0$ and $u_{\delta}=\tilde{u}_{\delta}$, which completes the proof.

\section{Coercivity of the quadratic form}

In this section we show Theorem 1.5.

Let $F$ be the $N+2$-dimensional space of the null directions for $Q$ that are generated by the continuous symmetries of the linear Schrödinger equation:

$$
F=\operatorname{span}_{\mathbb{C}}\left\{G_{0}, x_{j} G_{0},|x|^{2} G_{0}\right\}
$$

( $j=1$ or $j=1,2$ in dimension 1 and 2 respectively).

We must show that there exists a constant $c>0$ such that

$$
\varphi \in F^{\perp} \Longrightarrow Q(\varphi) \geq c\|\varphi\|_{L^{2}}^{2}
$$

It turns out that $F$ is generated by eigenfunctions for the harmonic oscillator defined in Section 5.1.1. Indeed, in dimension $1, F$ is spanned by $h_{0}, h_{1}$ and $h_{2}$ and in dimension 2 by $h_{00}, h_{10}, h_{01}$ and $h_{20}+h_{02}$.

The outline of this section is as follows. In Section 5.1 we recall some properties of the harmonic oscillator $\mathcal{H}=-\Delta+|x|^{2}$ and of a lens transform that will be used in the proof. In Section 5.2 we show that the proof of Theorem 1.5 reduces to the proof that $Q(\varphi)>0$ for any eigenfunction $\varphi$ of the harmonic oscillator $\mathcal{H}$ that is orthogonal to $F$. In Section 5.3 and Section 5.4 we treat the reduced problem in $1 D$ and $2 D$ respectively by estimating the values taken by the quadratic form on the eigenfunctions of $\mathcal{H}$. 


\subsection{Preliminaries}

\subsubsection{Harmonic oscillator}

Consider the linear Schrödinger equation with the harmonic potential:

$$
\begin{gathered}
i \partial_{\tau} u-\frac{1}{2} \mathcal{H} u=0, \quad(\tau, y) \in \mathbb{R} \times \mathbb{R}^{N}, \\
\text { where } \mathcal{H}=-\Delta+|y|^{2} .
\end{gathered}
$$

In what follows we briefly recall spectral property of $\mathcal{H}$. We refer to [5] and references therein for more details.

We first review the spectral properties of $\mathcal{H}$ in one space dimension. The spectrum of $\mathcal{H}$ consists of positive eigenvalues $\lambda_{n}=2 n+1, n=0,1, \ldots$, and the corresponding eigenfunctions are

$$
h_{n}(y)=(-1)^{n} c_{n} e^{y^{2} / 2} \partial_{y}^{n}\left(e^{-y^{2}}\right), \quad c_{n}=\frac{1}{\sqrt{n !} 2^{n / 2}},
$$

here the coefficients $c_{n}$ are chosen so that $\left\|h_{n}\right\|_{L^{2}(\mathbb{R})}^{2}=\sqrt{\pi}$. Equivalently, these are the Hermite functions

$$
h_{n}(y)=\frac{H_{n}(y)}{\sqrt{2^{n} n !}} e^{-y^{2} / 2},
$$

with $H_{n}(y)$ being the $n^{\text {th }}$ Hermite polynomial:

$$
H_{n}(y)=(-1)^{n} e^{y^{2}} \partial_{y}^{n}\left(e^{-y^{2}}\right) .
$$

Thus, $H_{0}(y)=1, H_{1}(y)=2 y, H_{2}(y)=4 y^{2}-2, H_{3}(y)=8 y^{3}-12 y, H_{4}(y)=$ $16 y^{4}-48 y^{2}+12$, etc. These eigenfunctions are orthogonal

$$
\int_{\mathbb{R}} h_{j}(y) h_{k}(y) d y=\frac{1}{\sqrt{2^{j} j !} \sqrt{2^{k} k !}} \int_{\mathbb{R}} H_{j}(y) H_{k}(y) e^{-y^{2}} d y=\sqrt{\pi} \delta_{j k},
$$

and they $\operatorname{span} L^{2}(\mathbb{R})$.

In the $2 D$ set up, $y=\left(y_{1}, y_{2}\right) \in \mathbb{R}^{2}$, the spectrum of $\mathcal{H}$ consists as well of a discrete set of positive eigenvalues $\left\{\lambda_{n}\right\}_{n \in \mathbb{N}}$ and, for $n \in \mathbb{N}$, one has

$$
\lambda_{n}=2 n+2 .
$$

To each eigenvalue $\lambda_{n}$ there corresponds a set of eigenfunctions $h_{j k}(y)$ with the property that $j+k=n$ and $h_{j k}(y)=h_{j}\left(y_{1}\right) h_{k}\left(y_{2}\right)$, where the $h_{n}$ 's are the onedimensional eigenfunctions. For example, $h_{00}(y)=e^{-|y|^{2}}$ is the only eigenfunction corresponding to the smallest eigenvalue $\lambda_{0}=2$. For $\lambda_{1}=4$, the eigenfunctions are

$$
h_{10}(y)=\sqrt{2} y_{1} e^{-|y|^{2} / 2} \text { and } h_{01}(y)=\sqrt{2} y_{2} e^{-|y|^{2} / 2} \text {, }
$$


for $\lambda_{2}=6$, they are

$$
\begin{gathered}
h_{20}(y)=2^{-1 / 2}\left(2 y_{1}^{2}-1\right) e^{-|y|^{2} / 2}, \quad h_{02}(y)=2^{-1 / 2}\left(2 y_{2}^{2}-1\right) e^{-|y|^{2} / 2} \\
\text { and } h_{11}(y)=2 y_{1} y_{2} e^{-|y|^{2} / 2} .
\end{gathered}
$$

\subsubsection{The Lens transform}

For a function $u(t, x): I \times \mathbb{R}^{N} \rightarrow \mathbb{C}$, define the lens transform ${ }^{1} \mathrm{~L} u$ of $u$ by

$$
\mathrm{Lu}(\tau, y)=\frac{1}{\cos ^{N / 2} \tau} u\left(\tan \tau, \frac{y}{\cos \tau}\right) e^{-i|y|^{2} \frac{\tan \tau}{2}} .
$$

The new variables $(\tau, y)$ are defined by $t=\tan \tau$ and $x=\frac{y}{\cos \tau}, \tau \in\left(-\frac{\pi}{2}, \frac{\pi}{2}\right)$, and thus, $\mathrm{L} u: \tan ^{-1}(I) \cap\left(-\frac{\pi}{2}, \frac{\pi}{2}\right) \times \mathbb{R}^{N} \rightarrow \mathbb{C}$. If $I=\mathbb{R}$, then $\mathrm{L} u:\left(-\frac{\pi}{2}, \frac{\pi}{2}\right) \times \mathbb{R}^{N} \rightarrow$ $\mathbb{C}$ : the lens transform compactifies the time. For more details see for example $[4,28]$ and reference therein.

If $u(t, x)$ solves (1.1) (for some $\gamma \in \mathbb{R}$ ), then $v=\mathrm{L} u(\tau, y)$ solves

$$
i \partial_{\tau} v-\frac{1}{2} \mathcal{H} v=-\gamma|v|^{\frac{4}{N}} v
$$

and vice versa.

The lens transform preserves the initial data $(\mathrm{Lu} u(0)=u(0)$, and thus, the mass of the solution:

$$
\|\left(\mathrm{Lu} u(0)\left\|_{L^{2}}=\right\| u(0) \|_{L^{2}} .\right.
$$

Furthermore, all Strichartz norms are also preserved, in particular:

$$
\|\mathrm{L} u\|_{L_{t, x}^{\frac{4}{N}+2}\left((-\pi / 2, \pi / 2) \times \mathbb{R}^{N}\right)}=\|u\|_{L_{t, x}^{\frac{4}{N}+2}\left(\mathbb{R} \times \mathbb{R}^{N}\right)} .
$$

Example 5.1. Let $G_{0}=\frac{1}{\pi^{N / 4}} e^{-|x|^{2} / 2}$. The solution to the linear Schrödinger equation (1.2) is given by (1.6). The definition of $\mathrm{L}$ shows that the solution $e^{-i \frac{\tau}{2} \mathcal{H}} G_{0}$ of (5.1) is given by

$$
\widetilde{G}(\tau, y)=\frac{1}{\pi^{N / 4}} e^{-i \frac{N}{2} \tau} e^{-|y|^{2} / 2}=(\mathrm{L} G)(\tau, y),
$$

which is consistent with the fact that $G_{0}$ is an eigenfunction for the eigenvalue $\lambda_{0}=N$ of $\mathcal{H}$ (in dimension $N=1,2$ ).

${ }^{1}$ We use the name 'lens transform' as in [28] but it should not be confused with the pseudoconformal inversion (1.5) of Talanov which is sometimes also called the lens transform. 
For later use we note that using the invariance of the initial condition and the $L^{\frac{4}{N}+2}$ norm by the lens transform $L$, we can rewrite the definition (3.3) of the quadratic form as

$$
\begin{aligned}
Q(\varphi)= & C_{S}\left[\frac{N+2}{N} \int|\varphi|^{2}+\frac{4(N+2)}{N^{2}}\left(\operatorname{Re} \int G_{0} \varphi\right)^{2}\right] \\
& -\frac{(N+2)^{2}}{N^{2}} \int_{-\frac{\pi}{2}}^{\frac{\pi}{2}} \int_{\mathbb{R}^{N}} G_{0}^{\frac{4}{N}}\left|e^{-i \frac{\tau}{2} \mathcal{H}} \varphi\right|^{2} \\
& -\frac{2(N+2)}{N^{2}} \operatorname{Re} \int_{-\frac{\pi}{2}}^{\frac{\pi}{2}} \int_{\mathbb{R}^{N}} G_{0}^{\frac{4}{N}} e^{i N \tau}\left(e^{-i \frac{\tau}{2} \mathcal{H}} \varphi\right)^{2} .
\end{aligned}
$$

\subsection{Reduction of the problem}

We prove here the following proposition:

Proposition 5.2. Assume that the conclusion of Theorem 1.5 does not hold. Then there exists an eigenfunction $\varphi$ of $\mathcal{H}$, satisfying the orthogonality relations (1.9) and such that $Q(\varphi)=0$.

We define

$$
E=\left\{\varphi \in L^{2}, \quad Q(\varphi)=0\right\} .
$$

Since $Q$ is a real positive quadratic form, we know that $E$ is a real vector space. Before proving Proposition 5.2, we need a few preliminary results.

Lemma 5.3. Let $\left\{\varphi_{n}\right\}$ be a bounded sequence in $L^{2}$ such that

$$
\lim _{n \rightarrow \infty} Q\left(\varphi_{n}\right)=0
$$

Then there exists a subsequence of $\left\{\varphi_{n}\right\}$ that converges strongly in $L^{2}$ to an element of $E$.

Proof. Assume after extraction,

$$
\varphi_{n} \rightarrow \varphi \text { weakly in } L^{2} \text { as } n \rightarrow \infty .
$$

Write

$$
Q(\varphi)=c_{Q} \int|\varphi|^{2}+B(\varphi, \varphi),
$$

where $c_{Q}=C_{S} \frac{N+2}{N}$ and the symmetric bilinear form $B$ is defined by

$$
\begin{aligned}
B(\varphi, \psi)= & C_{S} \frac{4(N+2)}{N^{2}}\left(\operatorname{Re} \int G_{0} \varphi\right)\left(\operatorname{Re} \int G_{0} \psi\right) \\
& -\frac{(N+2)^{2}}{N^{2}} \operatorname{Re} \int_{\mathbb{R}} \int_{\mathbb{R}^{N}}|G|^{\frac{4}{N}}\left(e^{i \frac{t}{2} \Delta} \varphi\right)\left(e^{-i \frac{t}{2} \Delta} \bar{\psi}\right) \\
& -\frac{2(N+2)}{N^{2}} \operatorname{Re} \int_{\mathbb{R}} \int_{\mathbb{R}^{N}} G^{\frac{4}{N}}\left(e^{i \frac{t}{2} \Delta} \varphi\right)\left(e^{i \frac{t}{2} \Delta} \psi\right) .
\end{aligned}
$$


We will use the following standard property of the Schrödinger linear flow:

\section{Claim 5.4.}

$$
\psi_{n} \rightarrow 0 \text { weakly in } L^{2} \Longrightarrow e^{i \frac{t}{2} \Delta} \psi_{n} \rightarrow 0 \text { strongly in } L_{\text {loc }}^{2}\left(\mathbb{R} \times \mathbb{R}^{N}\right) .
$$

Indeed, by the local smoothing effect $[7,23,32], e^{i \frac{t}{2} \Delta}$ defines a continuous map from $L^{2}\left(\mathbb{R}^{N}\right)$ to $L^{2}\left(\mathbb{R}, H_{\text {loc }}^{1 / 2}\left(\mathbb{R}^{N}\right)\right)$. Using the equation (1.2), we see that it also defines a continuous map from $L^{2}\left(\mathbb{R}^{N}\right)$ to $H_{\mathrm{loc}}^{1 / 4}\left(\mathbb{R}^{N+1}\right)$. The claim follows from the local compactness of the embedding of $H^{1 / 4}$ in $L^{2}$.

Combining Claim 5.4 with the decay of $G$ at infinity, we get

$$
\psi_{n} \rightarrow 0 \text { weakly in } L^{2} \Longrightarrow B\left(\psi_{n}, \psi_{n}\right) \rightarrow 0 .
$$

We will show by contradiction that $\left\{\varphi_{n}\right\}$ is a Cauchy sequence in $L^{2}$. If not, there exist sequences of integer $\left\{j_{n}\right\},\left\{k_{n}\right\}$ that go to $\infty$ such that

$$
\forall n, \quad\left\|\varphi_{k_{n}}-\varphi_{j_{n}}\right\|_{L^{2}} \geq \varepsilon_{0}>0 .
$$

The weak convergence of $\left\{\varphi_{n}\right\}$ in $L^{2}$ implies

$$
\varphi_{k_{n}}-\varphi_{j_{n}} \rightarrow 0 \text { weakly in } L^{2} .
$$

Furthermore, (5.7) and Cauchy-Schwarz inequality ( $Q$ is positive) implies

$$
0 \leq Q\left(\varphi_{j_{n}}-\varphi_{k_{n}}\right) \leq 2\left(Q\left(\varphi_{j_{n}}\right)+Q\left(\varphi_{k_{n}}\right)\right) \longrightarrow 0 \text { as } n \rightarrow \infty .
$$

Combining with (5.9) and (5.11) one gets

$$
\lim _{n \rightarrow \infty}\left\|\varphi_{j_{n}}-\varphi_{k_{n}}\right\|_{L^{2}}=0,
$$

contradicting (5.10). The proof is complete.

Lemma 5.5. The space $E$ is a finite dimensional vector space over $\mathbb{C}$.

Proof. The space $E$ is a vector space over $\mathbb{R}$. To show that it is a vector space over $\mathbb{C}$, it is sufficient to show that it is stable by multiplication by $i$. Let $\varphi \in E$. Write $\varphi=\alpha G_{0}+\tilde{\varphi}$, with $\alpha=\int \varphi G_{0}$, so that

$$
\int \tilde{\varphi} G_{0}=0 .
$$

The function $i \alpha G$ is in $E$ and $E$ is stable by addition. To show that $i \varphi \in E$ we must show that $i \tilde{\varphi} \in E$. By (5.6),

$$
\begin{aligned}
Q(i \tilde{\varphi})= & Q(\tilde{\varphi})-\frac{8(N+2)}{N^{2}} C_{S}\left(\operatorname{Re} \int G_{0} \tilde{\varphi}\right)^{2} \\
& +\frac{4(N+2)}{N} \operatorname{Re} \int_{-\pi / 2}^{\pi / 2} \int_{\mathbb{R}^{N}} G_{0}^{\frac{4}{N}} e^{i N \tau}\left(e^{-i \frac{\tau}{2} \mathcal{H}} \tilde{\varphi}\right)^{2} .
\end{aligned}
$$


We know that $\tilde{\varphi} \in E$, so $Q(\tilde{\varphi})=0$ and it suffices to show:

$$
\begin{gathered}
\left(\operatorname{Re} \int G_{0} \tilde{\varphi}\right)^{2}=0 \\
\operatorname{Re} \int_{-\pi / 2}^{\pi / 2} \int_{\mathbb{R}^{N}} G_{0}^{\frac{4}{N}} e^{i N \tau}\left(e^{-i \frac{\tau}{2} \mathcal{H}} \tilde{\varphi}\right)^{2}=0 .
\end{gathered}
$$

The first equality follows immediately from (5.12). Let us show the second equality in the case $N=2$. By (5.12), $\tilde{\varphi}$ is orthogonal to the first eigenfunction $h_{00}$ of $\mathcal{H}$. Thus, $e^{-i \frac{\tau}{2} \mathcal{H}} \tilde{\varphi}$ is of the form

$$
e^{-i \frac{\tau}{2} \mathcal{H}} \tilde{\varphi}=\sum_{\substack{\left(n_{1}, n_{2}\right) \in \mathbb{N}^{2} \\ n_{1}+n_{2} \geq 1}} \alpha_{n_{1} n_{2}} e^{-i \tau\left(n_{1}+n_{2}+1\right)} h_{n_{1} n_{2}}(y),
$$

where by definition $\alpha_{n_{1} n_{2}}=\int_{\mathbb{R}^{2}} \tilde{\varphi}(y) h_{n_{1} n_{2}}(y) d y$. It follows from the definition of $h_{n_{1} n_{2}}$ that it is even if $n_{1}+n_{2}$ is even and odd if $n_{1}+n_{2}$ is odd. Expanding $\left(e^{-i \frac{\tau}{2} \mathcal{H}} \tilde{\varphi}\right)^{2}$, we can write

$$
\operatorname{Re} \int_{-\pi / 2}^{\pi / 2} \int_{\mathbb{R}^{2}} G_{0}^{2} e^{2 i \tau}\left(e^{-i \frac{\tau}{2} \mathcal{H}} \tilde{\varphi}\right)^{2}=\operatorname{Re} \int_{-\pi / 2}^{\pi / 2} \int_{\mathbb{R}^{2}} G_{0}^{2} e^{2 i \tau} \sum_{m \geq 4} e^{-i \tau m} g_{m}(y) d y d \tau
$$

where $m \geq 4$ and $g_{m} \in C^{\infty}\left(\mathbb{R}^{N}\right)$ is exponentially decaying. Again, $g_{m}$ is even if $m$ is even and odd if $m$ is odd. Then (5.14) will follow from

$$
\operatorname{Re} \int_{-\pi / 2}^{\pi / 2} \int_{\mathbb{R}^{2}} G_{0}^{2}(x) e^{i 2 \tau} e^{-i \tau m} g_{m}(y) d y d \tau=0 .
$$

We distinguish two cases. If $m$ is odd, then $\int_{\mathbb{R}^{2}} G_{0}(y)^{2} g_{m}(y) d y=0$ (it is the integral of an odd function on $\mathbb{R}^{2}$ ), and (5.15) follows. If $m$ is even, using that $m \geq$ 4 , we get that $\int_{-\pi / 2}^{\pi / 2} e^{2 i \tau-i \tau m} d \tau=0$, which implies also (5.15). This completes the proof of (5.14) in the case $N=2$. To prove (5.14) in the case $N=1$ write

$$
e^{-i \frac{\tau}{2} \mathcal{H}} \tilde{\varphi}_{0}=\sum_{n \geq 1} \alpha_{n} e^{-i \tau\left(n+\frac{1}{2}\right)} h_{n}(y)
$$

and argue as above. We leave the details to the reader.

It follows immediately from Lemma 5.3 that the unit ball of $\left(E,\|\cdot\|_{L^{2}}\right)$ is compact, concluding the proof of Lemma 5.5.

We next prove Proposition 5.2. Let $\widetilde{E}=F^{\perp} \cap E$. By definition, $\widetilde{E}$ is the subspace of functions $\varphi \in L^{2}$ satisfying $Q(\varphi)=0$ and the orthogonality relations (1.9). By Lemma 5.5 it is a complex, finite dimensional vector space. 
We argue by contradiction, assuming that the conclusion of Theorem 1.5 does not hold.

Step 1. Existence of a nontrivial null-space for $Q$. In this step we show that the negation of Theorem 1.5 implies that $\widetilde{E}$ is not reduced to $\{0\}$. Indeed, in this case, there exists a sequence $\varphi_{n}$ in $L^{2}$ such that

$$
\forall n, \quad \varphi_{n} \in F^{\perp} \text { and } n Q\left(\varphi_{n}\right)<\left\|\varphi_{n}\right\|_{L^{2}}=1 .
$$

By Lemma 5.3, a subsequence of $\left\{\varphi_{n}\right\}_{n}$ converges strongly in $L^{2}$ to some $\psi \in E$. The condition $\left\|\varphi_{n}\right\|_{L^{2}}=1$ implies that $\|\psi\|_{L^{2}}=1$ and, in particular, that $\psi \neq 0$. Furthermore, $\varphi_{n} \in F^{\perp}$ for all $n$ and $F^{\perp}$ is closed, thus, $\psi \in F^{\perp}$, which shows as announced that $\operatorname{dim} \widetilde{E} \geq 1$.

Step 2. Stability by the harmonic evolution. In this step we show that $\widetilde{E}$ is invariant by $e^{-i \frac{\tau_{0}}{2} \mathcal{H}}$ for any $\tau_{0} \in \mathbb{R}$. As $\widetilde{E}$ is a complex vector space, it is equivalent to show that $\widetilde{E}$ is invariant by $S\left(t_{0}\right)=e^{-i \frac{\mathcal{H}-N}{2} \tau_{0}}$. The space $F$ admits a basis of eigenfunctions of $\mathcal{H}$, thus $F^{\perp}$ is stable by $S\left(\tau_{0}\right)$. To prove that $E$ is stable by $S\left(\tau_{0}\right)$, we rewrite the equation (3.2) using the lens transform of Section 5.1.2

$$
\begin{aligned}
C_{S}\left(\int_{\mathbb{R}^{N}}\left|G_{0}+\varphi\right|^{2}\right)^{1+\frac{2}{N}} & -\int_{-\pi / 2}^{\pi / 2} \int_{\mathbb{R}^{N}}\left|e^{-i \frac{N \tau}{2}} G_{0}+e^{-i \frac{\tau}{2} \mathcal{H}} \varphi\right|^{2+\frac{4}{N}} d y d \tau \\
& =Q(\varphi)+\mathcal{O}\left(\|\varphi\|_{L^{2}}^{3}\right) .
\end{aligned}
$$

We will show that the two terms in the first line of (5.17) do not change when replacing $\varphi$ by $S\left(\tau_{0}\right) \varphi$, which will imply that

$$
Q\left(S\left(\tau_{0}\right) \varphi\right)=Q(\varphi),
$$

and thus, that $E$ and $\widetilde{E}=E \cap F$ are stable by $S\left(\tau_{0}\right)$ ).

By mass conservation

$$
\begin{aligned}
\int_{\mathbb{R}^{N}}\left|G_{0}+S\left(\tau_{0}\right) \varphi\right|^{2} & =\int_{\mathbb{R}^{N}}\left|e^{-i \frac{N \tau_{0}}{2}} G_{0}+e^{-i \frac{\tau_{0}}{2} \mathcal{H}} \varphi\right|^{2} \\
& =\int\left|e^{-i \frac{\tau_{0}}{2} \mathcal{H}}\left(G_{0}+\varphi\right)\right|^{2}=\int\left|G_{0}+\varphi\right|^{2} .
\end{aligned}
$$

Similarly,

$$
\begin{gathered}
\int_{-\pi / 2}^{\pi / 2} \int_{\mathbb{R}^{N}}\left|e^{-i \frac{N \tau}{2}} G_{0}+e^{-i \frac{\tau}{2} \mathcal{H}} S\left(\tau_{0}\right) \varphi\right|^{2+\frac{4}{N}} \\
=\int_{-\pi / 2+\tau_{0}}^{\pi / 2+\tau_{0}} \int_{\mathbb{R}^{N}}\left|e^{-i \frac{\tau}{2} \mathcal{H}}\left(G_{0}+\varphi\right)\right|^{2+\frac{4}{N}} \\
=\int_{-\pi / 2}^{\pi / 2} \int_{\mathbb{R}^{N}}\left|e^{-i \frac{\tau}{2} \mathcal{H}}\left(G_{0}+\varphi\right)\right|^{2+\frac{4}{N}} .
\end{gathered}
$$


The last equality is consequence of the following known identity (see e.g. equality (2.5) in [5]), which can be easily checked by expanding $\varphi$ in the Hilbert basis of $L^{2}$ given by the eigenfunctions of $\mathcal{H}$ :

$$
e^{-i \frac{\pi+\tau}{2} \mathcal{H}} \varphi(y)=e^{-i N \frac{\pi}{2}} e^{-i \frac{\tau}{2} \mathcal{H}} \varphi(-y) .
$$

This concludes the proof of (5.18).

Step 3. End of the proof. We have shown that $e^{-i \frac{\tau}{2} \mathcal{H}}$ is a strongly continuous group of operators on the finite dimensional vector space $\widetilde{E}$. As a consequence, $e^{-i \frac{\tau}{2} \mathcal{H}}=e^{\tau A}$ for some $A \in \mathcal{L}(\widetilde{E})$ (see for example [10, Theorem 2.9, page 11]).

Let $f \in \widetilde{E}$. Then

$$
\lim _{\tau \rightarrow 0} \frac{e^{-i \frac{\tau}{2} \mathcal{H}} f-f}{\tau}=\lim _{\tau \rightarrow 0} \frac{e^{\tau A} f-f}{\tau}=A f .
$$

This shows that $f$ is in the domain of $\mathcal{H}$ and that $A f=-\frac{i}{2} \mathcal{H} f$. As a consequence, $\mathcal{H}=2 i A$ is a continuous linear operator on $\widetilde{E}$. Using that $\widetilde{E}$ is finite dimensional, we deduce that $\mathcal{H}$ admits an eigenfunction in $\widetilde{E}$, concluding the proof of Proposition 5.2.

From now on we treat each dimension separately.

\subsection{D case}

In this case, the quadratic form is

$$
\begin{aligned}
Q(\varphi)= & \sqrt{3} \int|\varphi|^{2} d y+\frac{4 \sqrt{3}}{\sqrt{\pi}}\left(\operatorname{Re} \int e^{-y^{2} / 2} \varphi(y) d y\right)^{2} \\
& -\frac{9}{\pi} \int_{-\frac{\pi}{2}}^{\frac{\pi}{2}} \int e^{-2 y^{2}}\left|e^{-i \frac{\tau}{2} \mathcal{H}} \varphi\right|^{2} d y d \tau \\
& -\frac{6}{\pi} \operatorname{Re} \int_{-\frac{\pi}{2}}^{\frac{\pi}{2}} \int e^{-2 y^{2}} e^{i \tau}\left(e^{-i \frac{\tau}{2} \mathcal{H}} \varphi\right)^{2} d y d \tau .
\end{aligned}
$$

Recall that $h_{0}$ is the 0th Hermite function (the eigenfunction corresponding to $\lambda_{0}=$ 1), and $e^{-i \frac{\tau}{2} \mathcal{H}} h_{0}=e^{-i \frac{\tau}{2}} e^{-y^{2} / 2}$. Similarly,

$$
h_{1}(y)=\sqrt{2} y e^{-y^{2} / 2} \rightsquigarrow e^{-i \frac{\tau}{2} \mathcal{H}} h_{1}(y)=\sqrt{2} e^{-\frac{3}{2} i \tau} y e^{-y^{2} / 2},
$$

and

$$
h_{2}(y)=\frac{1}{\sqrt{2}}\left(2 y^{2}-1\right) e^{-y^{2} / 2} \rightsquigarrow \quad e^{-i \frac{\tau}{2} \mathcal{H}} h_{2}(y)=\frac{1}{\sqrt{2}} e^{-\frac{5}{2} i \tau}\left(2 y^{2}-1\right) e^{-y^{2} / 2},
$$


then it is easy to check that

$$
Q\left(h_{0}\right)=Q\left(i h_{0}\right)=Q\left(h_{1}\right)=Q\left(i h_{1}\right)=Q\left(h_{2}\right)=Q\left(i h_{2}\right)=0 .
$$

Note that for the rest of $h_{j}, j \geq 3$, we have $e^{-i \frac{\tau}{2} \mathcal{H}} h_{j}=e^{-i(2 j+1) \frac{\tau}{2}} h_{j}$, and when computing the quadratic form $Q\left(h_{j}\right)$, we obtain that by orthogonality of $\left\{h_{j}\right\}$ the second term in (5.20) is zero. Integration in $t$ over the full circle makes the fourth term vanish, therefore producing

$$
Q\left(h_{j}\right)=\sqrt{3} \int\left|h_{j}(y)\right|^{2} d y-9 \int e^{-2 y^{2}}\left|h_{j}(y)\right|^{2} d y .
$$

Since $e^{-2 y^{2}}$ is dominated by $e^{-y^{2}}$, we estimate the second term by

$$
\int e^{-y^{2}}\left|h_{j}(y)\right|^{2} d y=\frac{(2 j) !}{2^{2 j}(j !)^{2}} \sqrt{\frac{\pi}{2}},
$$

(see [33, Lemma 2.1]). Then, using the following estimate for the central binomial coefficient

$$
\left(\begin{array}{c}
2 m \\
m
\end{array}\right) \leq \frac{4^{m}}{\sqrt{3 m+1}}, \quad m \geq 1,
$$

we obtain

$$
\begin{aligned}
Q\left(h_{j}\right) & \geq \sqrt{3 \pi}\left(1-3 \sqrt{\frac{3}{2}} \frac{(2 j) !}{2^{2 j}(j !)^{2}}\right) \\
& \geq \sqrt{3 \pi}\left(1-\frac{3 \sqrt{3}}{\sqrt{2} \sqrt{3 j+1}}\right)>0,
\end{aligned}
$$

for $j>4$. Explicit computation shows that

$$
Q\left(h_{3}\right)=\frac{2 \sqrt{\pi}}{3 \sqrt{3}} \quad \text { for } \quad h_{3}(y)=\frac{1}{\sqrt{3}}\left(2 y^{3}-3 y\right) e^{-y^{2} / 2}
$$

and

$$
Q\left(h_{4}\right)=\frac{8 \sqrt{\pi}}{9 \sqrt{3}} \text { for } h_{4}(y)=\frac{1}{2 \sqrt{6}}\left(4 y^{4}-12 y^{2}+3\right) e^{-y^{2} / 2},
$$

concluding the proof that $Q\left(h_{j}\right)>0$ for all $j \geq 3$.

\section{4. $2 \mathrm{D}$ case}

Recall from Section 5.1.1 the definitions of the basis $h_{j k}$ of eigenfunctions of $\mathcal{H}$. By definition $h_{j k}(y)=h_{j}\left(y_{1}\right) h_{k}\left(y_{2}\right)$, where $\left\{h_{j}\right\}_{j \geq 0}$ is the orthogonal system in $L^{2}(\mathbb{R})$ of eigenfunctions of the $1 D$ harmonic oscillator. The function $h_{j k}$ corresponds to the eigenvalue $\lambda_{m}$ with $m=j+k$, and $\lambda_{m}=2 m+2=2(j+k)+2$. For 
a fixed $m$ there are $m+1$ independent eigenfunctions $h_{j k} \equiv h_{j, m-j}, 0 \leq j \leq m$, corresponding to $\lambda_{m}$. The space $F$ is exactly

$$
F=\operatorname{span}_{\mathbb{C}}\left\{h_{00}, h_{01}, h_{10}, h_{02}+h_{20}\right\} .
$$

By Proposition 5.2, the proof of Theorem 1.5 in $2 D$ is reduced to the following:

Proposition 5.6. Assume that $N=2$. Then

$$
\begin{array}{r}
\text { If } \alpha \neq \beta \text { or } \gamma \neq 0, \quad Q\left(\alpha h_{02}+\beta h_{20}+\gamma h_{11}\right)>0 . \\
\text { If } m \geq 3 \text { and } \sum_{j=0}^{m}\left|\alpha_{j}\right|^{2} \neq 0, \text { then } Q\left(\sum_{j=0}^{m} \alpha_{j} h_{j, m-j}\right)>0 .
\end{array}
$$

Proof. Let $\varphi \in L^{2}$. By (5.6) with $N=2$, we have

$$
\begin{aligned}
Q(\varphi)= & \int_{\mathbb{R}^{2}}|\varphi|^{2}+2\left(\operatorname{Re} \int G_{0} \varphi\right)^{2} \\
& -4 \int_{-\pi / 2}^{\pi / 2} \int_{\mathbb{R}^{2}} G_{0}^{2}\left|e^{-i \frac{\tau}{2} \mathcal{H}} \varphi\right|^{2} \\
& -2 \operatorname{Re} \int_{-\pi / 2}^{\pi / 2} \int_{\mathbb{R}^{2}} G_{0}^{2} e^{i 2 \tau}\left(e^{-i \frac{\tau}{2} \mathcal{H}} \varphi\right)^{2} .
\end{aligned}
$$

It is easy to check that $Q\left(h_{00}\right)=0$.

Let $m \geq 1$. Any eigenfunction of $\mathcal{H}$ for the eigenvalue $2 m+2$ is of the form

$$
\varphi=\sum_{j=0}^{m} \alpha_{j} h_{j, m-j} .
$$

If $\varphi$ is of this form, then the second integral in $Q(\varphi)$ vanishes because of the orthogonality of the $h_{j k}$ 's and so does the last term, since $\int_{-\pi / 2}^{\pi / 2} e^{i 2 m t} d t=0$ as $m \in \mathbb{N} \backslash\{0\}$.

Recall that the first eigenfunction for $\mathcal{H}$ is $h_{00}(y)=e^{-\frac{1}{2}|y|^{2}}$. Using that $G_{0}=$ $\frac{1}{\sqrt{\pi}} e^{-\frac{|y|^{2}}{2}}$, we obtain

$$
Q(\varphi)=\mathcal{B}(\varphi, \varphi), \quad \mathcal{B}(\varphi, \psi)=\operatorname{Re} \int_{\mathbb{R}^{2}} \varphi \bar{\psi}-4 \operatorname{Re} \int_{\mathbb{R}^{2}} h_{00}^{2} \varphi \bar{\psi}
$$

In particular, if $j+k \geq 1$,

$$
\begin{aligned}
Q\left(h_{j k}\right)= & \left(\int h_{j}^{2}\left(y_{1}\right) d y_{1}\right)\left(\int h_{k}^{2}\left(y_{2}\right) d y_{2}\right) \\
& -4\left(\int e^{-y_{1}^{2}} h_{j}^{2}\left(y_{1}\right) d y_{1}\right)\left(\int e^{-y_{2}^{2}} h_{k}^{2}\left(y_{2}\right) d y_{2}\right) \\
= & \pi\left(1-\frac{(2 j) !(2 k) !}{2^{2(j+k)-1}(j !)^{2}(k !)^{2}}\right),
\end{aligned}
$$


where in the last line we used the product of Hermite functions from [33, Lemma 2.1]. As expected we get $Q\left(h_{01}\right)=Q\left(h_{10}\right)=0$.

Define

$$
G(j, k)= \begin{cases}\frac{(j+k) !}{2^{(j+k)-1 / 2} \sqrt{j !} \sqrt{k !}\left(\frac{j+k}{2}\right) !} & \text { for } j+k-\text { even } \\ 0 & \text { for } j+k-\text { odd }\end{cases}
$$

For a product of two $G$ functions, write

$$
F(m, j, k)=G(j, k) G(m-j, m-k) .
$$

Observe that $F$ is symmetric, i.e.,

$$
F(m, j, k)=F(m, k, j)=F(m, m-j, m-k)=F(m, m-k, m-j) .
$$

Note as well that

$Q\left(h_{j, m-j}\right)=\pi(1-F(m, j, j)), \quad j \neq k \Longrightarrow \mathcal{B}\left(h_{j, m-j}, h_{k, m-k}\right)=\pi F(m, j, k)$,

and that for $\alpha, \beta, \gamma \in \mathbb{C}$

$$
\frac{1}{\pi} Q\left(\alpha h_{02}+\beta h_{20}+\gamma h_{11}\right)=\frac{1}{4}|\alpha-\beta|^{2}+\frac{1}{2}|\gamma|^{2},
$$

which is equal to zero if and only if $\alpha=\beta$ and $\gamma=0$. This shows (5.22).

Let us show (5.23).

We have

$$
\begin{aligned}
& \frac{1}{\pi} Q\left(\sum_{j=0}^{m} \alpha_{j} h_{j, m-j}\right) \\
& \quad=\sum_{j=0}^{m}\left|\alpha_{j}\right|^{2}(1-F(m, j, j))-2\left(\operatorname{Re} \sum_{\substack{j<k, j+k-\text { even }}} \alpha_{j} \bar{\alpha}_{k} F(m, j, k)\right) \\
& \quad \geq \sum_{j=0}^{m}\left|\alpha_{j}\right|^{2}-\left(\sum_{j=0}^{m}\left|\alpha_{j}\right|^{2} F(m, j, j)+\sum_{\substack{j<k, j+k-\text { even }}}\left(\left|\alpha_{j}\right|^{2}+\left|\alpha_{k}\right|^{2}\right) F(m, j, k)\right) \\
& \geq \sum_{j=0}^{m}\left|\alpha_{j}\right|^{2}\left(1-\sum_{\substack{k \in[0, m], j+k-\text { even }}} F(m, j, k)\right)
\end{aligned}
$$


where we used the symmetry of $F$ in the last line. By Cauchy-Schwarz, for any $j \in[0, m]$ we obtain

$$
\begin{aligned}
\mathcal{F}(m, j) & :=\sum_{\substack{k \in[0, m], j+k-\text { even }}} F(m, j, k) \\
& =\frac{2}{2^{2 m}} \sum_{\substack{k \in[0, m], j+k-\text { even }}} \frac{(j+k) !(2 m-(j+k)) !}{\sqrt{j ! k !(m-j) !(m-k) !}\left(\frac{j+k}{2}\right) !\left(m-\frac{j+k}{2}\right) !} \\
& \leq \frac{2}{2^{2 m}}\left(\sum_{\substack{k \in[0, m], j+k-\text { even }}}\left(\begin{array}{c}
j+k \\
k
\end{array}\right)\left(\begin{array}{c}
2 m-(j+k) \\
m-k
\end{array}\right)\right)^{1 / 2} \\
& \left.\times \sum_{\substack{k \in[0, m], j+k-\text { even }}}\left(\begin{array}{c}
j+k \\
\frac{j+k}{2}
\end{array}\right)\left(\begin{array}{c}
2 m-(j+k) \\
m-\frac{j+k}{2}
\end{array}\right)\right)^{1 / 2} \\
& \leq \frac{2}{4^{m}} \mathrm{I} \times \text { II. }
\end{aligned}
$$

By elementary combinatorial arguments (see Appendix D) and (5.21), we estimate the term I

$$
\begin{aligned}
\mathrm{I}^{2} & \leq \frac{1}{2}\left[\left(\begin{array}{c}
2 m+1 \\
m+1
\end{array}\right)+\left(\begin{array}{c}
2 m \\
m
\end{array}\right)\right]=\frac{1}{2}\left[\frac{m+1}{2 m+2}\left(\begin{array}{c}
2 m+2 \\
m+1
\end{array}\right)+\left(\begin{array}{c}
2 m \\
m
\end{array}\right)\right] \\
& <\frac{1}{2}\left[\frac{1}{2} \frac{4^{m+1}}{\sqrt{3(m+1)+1}}+\frac{4^{m}}{\sqrt{3 m+1}}\right] \\
& =4^{m}\left(\frac{1}{\sqrt{3 m+4}}+\frac{1}{2 \sqrt{3 m+1}}\right) .
\end{aligned}
$$

For the term II we use (5.21), then decompose into fractions:

$$
\begin{aligned}
\mathrm{II}^{2} & \leq 4^{m} \sum_{\substack{k \in[0, m], j+k-\text { even }}} \frac{1}{\sqrt{3\left(\frac{j+k}{2}\right)+1}} \frac{1}{\sqrt{3\left(m-\frac{j+k}{2}\right)+1}} \\
& =\frac{4^{m}}{\sqrt{3 m+2}} \sum_{\substack{k \in[0, m], j+k-\text { even }}}\left(\frac{1}{3\left(\frac{j+k}{2}\right)+1}+\frac{1}{3\left(m-\frac{j+k}{2}\right)+1}\right)^{1 / 2} .
\end{aligned}
$$

Using the inequality $\sqrt{a+b} \leq \sqrt{a}+\sqrt{b}$, reindexing the summation and estimating 
the sum we obtain

$$
\begin{aligned}
\mathrm{II}^{2} & \leq \frac{4^{m}}{\sqrt{3 m+2}} \sum_{l=0}^{[m / 2]}\left(\frac{1}{\sqrt{3 l+1}}+\frac{1}{\sqrt{3(m-l)+1}}\right) \\
& =\frac{4^{m}}{\sqrt{3 m+2}}\left(\sum_{l=0}^{m} \frac{1}{\sqrt{3 l+1}}+\frac{1}{\sqrt{3 \frac{m}{2}+1}} \chi_{\{m-\text { even }\}}\right) \\
& \leq \frac{4^{m}}{\sqrt{3 m+2}}\left(\frac{2}{3}(\sqrt{3 m+1}-1)+1+\frac{1}{\sqrt{1.5 m+1}} \chi_{\{m-\text { even }\}}\right),
\end{aligned}
$$

where $\chi_{\{m-\text { even }\}}=1$ if $m$ is even, 0 if $m$ is odd. Hence,

$$
\begin{aligned}
\mathcal{F}(m, j) \leq 2 & {\left[\left(\frac{1}{\sqrt{3 m+4}}+\frac{1}{2 \sqrt{3 m+1}}\right)\right.} \\
& \left.\times \frac{1}{\sqrt{3 m+2}}\left(\frac{2 \sqrt{3 m+1}+1}{3}+\frac{1}{\sqrt{1.5 m+1}} \chi_{\{m-\text { even }\}}\right)\right]^{1 / 2}
\end{aligned}
$$

\begin{tabular}{|c|c|c|c|c|c|c|c|}
\hline \multirow{2}{*}{$m=3$} & $\mathcal{F}(3,0)$ & $\mathcal{F}(3,1)$ & $\mathcal{F}(3,2)$ & $\mathcal{F}(3,3)$ & & & \\
\hline & 0.841 & 0.591 & 0.591 & 0.841 & & & \\
\hline \multirow[t]{2}{*}{$m=4$} & $\mathcal{F}(4,0)$ & $\mathcal{F}(4,1)$ & $\mathcal{F}(4,2)$ & $\mathcal{F}(4,3)$ & $\mathcal{F}(4,4)$ & & \\
\hline & 0.785 & 0.5 & 0.664 & 0.5 & 0.785 & & \\
\hline \multirow[t]{2}{*}{$m=5$} & $\mathcal{F}(5,0)$ & $\mathcal{F}(5,1)$ & $\mathcal{F}(5,2)$ & $\mathcal{F}(5,3)$ & $\mathcal{F}(5,4)$ & $\mathcal{F}(5,5)$ & \\
\hline & 0.718 & 0.492 & 0.573 & 0.573 & 0.492 & 0.718 & \\
\hline \multirow[t]{2}{*}{$m=6$} & $\mathcal{F}(6,0)$ & $\mathcal{F}(6,1)$ & $\mathcal{F}(6,2)$ & $\mathcal{F}(6,3)$ & $\mathcal{F}(6,4)$ & $\mathcal{F}(6,5)$ & $\mathcal{F}(6,6)$ \\
\hline & 0.673 & 0.454 & 0.563 & 0.495 & 0.563 & 0.454 & 0.673 \\
\hline
\end{tabular}

which is less than 1 for $m \geq 7$. For $m=3,4,5,6$ we provide the values of $\mathcal{F}(m, j)$ in Table 5.1 (which are all smaller than 1).

Table 5.1. Values of $\mathcal{F}(m, j)$ for $3 \leq m \leq 6$.

\section{Appendix}

\section{A. Implicit function theorem and orthogonality conditions}

In this appendix we prove Claim 3.5. By explicit computation,

$$
\nabla G_{0}=-x G_{0}, \quad \Delta G_{0}=\left(|x|^{2}-N\right) G_{0} .
$$


The preceding identities imply that at the point $\left(0, \Gamma_{i d}, G_{0}\right)$ :

$$
\begin{gathered}
\frac{\partial U_{\delta}}{\partial \theta_{0}}=-i \delta G_{0}, \quad \frac{\partial U_{\delta}}{\partial \rho_{0}}=-\frac{N}{2} \delta G_{0}-\delta x \cdot \nabla G_{0}=-\frac{N}{2} \delta G_{0}+\delta|x|^{2} G_{0}, \\
\frac{\partial U_{\delta}}{\partial \xi_{0}}=-i \delta x G_{0}, \quad \frac{\partial U_{\delta}}{\partial x_{0}}=-\nabla G_{0}=\delta x G_{0}, \\
\frac{\partial U_{\delta}}{\partial t_{0}}=-\frac{i}{2} \delta \Delta G_{0}-i \gamma \delta^{\frac{4}{N}+1}\left|G_{0}\right|^{\frac{4}{N}} G_{0}=\frac{i}{2} \delta\left(N-|x|^{2}\right) G_{0}-i \gamma \delta^{\frac{4}{N}+1}\left|G_{0}\right|^{\frac{4}{N}} G_{0} .
\end{gathered}
$$

Using the equalities

$$
\int G_{0}^{2}=1, \quad \int|x|^{2} G_{0}^{2}=\frac{N}{2}, \quad \int|x|^{4} G_{0}^{2}=\frac{N(N+2)}{4},
$$

which follow from the normalization of $G_{0}$ and (A.1), we get that the Jacobian $\left(\frac{\partial \Phi_{\delta}^{k}}{\partial \theta_{0}}, \frac{\partial \Phi_{\delta}^{k}}{\partial \rho_{0}}, \frac{\partial \Phi_{\delta}^{k}}{\partial \xi_{0}}, \frac{\partial \Phi_{\delta}^{k}}{\partial x_{0}}, \frac{\partial \Phi_{\delta}^{k}}{\partial t_{0}}\right)_{k=1 \ldots 5}$ of $\Phi_{\delta}$ with respect to the variables $\left(\theta_{0}, \rho_{0}, \xi_{0}, x_{0}, t_{0}\right)$ at the point $\left(0, \Gamma_{i d}, G_{0}\right)$ is of the form

$$
\left(\begin{array}{ccccc}
-1 & 0 & 0 & 0 & \frac{1}{4}+\mathcal{O}\left(\delta^{4}\right) \\
0 & \frac{1}{2} & 0 & 0 & 0 \\
0 & 0 & -\frac{1}{2} & 0 & 0 \\
0 & 0 & 0 & \frac{1}{2} & 0 \\
0 & 0 & 0 & 0 & -\frac{1}{4}+\mathcal{O}\left(\delta^{4}\right)
\end{array}\right), \quad\left(\begin{array}{ccccccc}
-1 & 0 & 0 & 0 & 0 & 0 & \frac{1}{2}+\mathcal{O}\left(\delta^{2}\right) \\
0 & 1 & 0 & 0 & 0 & 0 & 0 \\
0 & 0 & -\frac{1}{2} & 0 & 0 & 0 & 0 \\
0 & 0 & 0 & -\frac{1}{2} & 0 & 0 & 0 \\
0 & 0 & 0 & 0 & \frac{1}{2} & 0 & 0 \\
0 & 0 & 0 & 0 & 0 & \frac{1}{2} & 0 \\
0 & 0 & 0 & 0 & 0 & 0 & -\frac{1}{2}+\mathcal{O}\left(\delta^{2}\right)
\end{array}\right)
$$

in dimensions $N=1$ or 2 respectively. Using that these matrices are invertible, and that their inverses may be estimated uniformly with respect to $\delta \in\left(0, \delta_{0}\right)\left(\delta_{0}\right.$ small), we deduce from the implicit functions theorem that there exists $\varepsilon>0$ and a constant $C>0$ such that for small $\delta$, if $\left\|f-G_{0}\right\|_{L^{2}}<\varepsilon$, there exists $\left(\theta_{\delta}, \rho_{\delta}, \xi_{\delta}, x_{\delta}, t_{\delta}\right)=$ $\left(\theta_{\delta}, \Gamma_{\delta}\right)$ such that

$$
\left|\theta_{\delta}\right|+\left|\rho_{\delta}-1\right|+\left|\xi_{\delta}\right|+\left|x_{\delta}\right|+\left|t_{\delta}\right| \leq C\left\|f-G_{0}\right\|_{L^{2}} \text { and } \Phi_{\delta}\left(\theta_{\delta}, \Gamma_{\delta}, f\right)=0
$$

Applying this to the family $\left\{\delta^{-1} g_{\delta}\right\}_{\delta}$ of Step 1 in the proof of Proposition 3.3, we get as announced that there exists $\left(\theta_{\delta}, \Gamma_{\delta}\right)=\left(\theta_{\delta}, \rho_{\delta}, \xi_{\delta}, x_{\delta}, t_{\delta}\right)$ such that

$$
\lim _{\delta \rightarrow \infty}\left|\theta_{\delta}\right|+\left|\rho_{\delta}-1\right|+\left|\xi_{\delta}\right|+\left|x_{\delta}\right|+\left|t_{\delta}\right|=0 \text { and } \Phi_{\delta}\left(\theta_{\delta}, \Gamma_{\delta}, \delta^{-1} g_{\delta}\right)=0
$$

concluding the proof. 


\section{B. Constant in 1D and the generating function trick}

By (3.16),

$$
D_{1}=6 \operatorname{Re} \iint|G(t)|^{4} \bar{G}(t) r(t) d t d x,
$$

where $r$ is the solution to

$$
i \partial_{t} r+\frac{1}{2} \Delta r+|G|^{4} G=0, \quad r(0, x)=0 .
$$

Let $\mathrm{L}$ be the lens transform defined in Section 5.1.2. By the change of variable $t=\tan \tau, x=\frac{y}{\cos \tau}, \tau \in(-\pi / 2, \pi / 2)$, we get

$$
D_{1}=6 \operatorname{Re} \int_{\mathbb{R}} \int_{-\pi / 2}^{\pi / 2}|\mathrm{~L} G|^{4} \overline{\mathrm{L} G} \mathrm{~L} r d \tau d y .
$$

By the example at the end of Section 5.1.2, $\mathrm{L} G=\frac{1}{\pi^{1 / 4}} e^{-i \tau / 2} e^{-y^{2} / 2}$, and thus,

$$
D_{1}=\frac{6}{\pi^{5 / 4}} \operatorname{Re} \int_{\mathbb{R}} \int_{-\pi / 2}^{\pi / 2} e^{-5 y^{2} / 2} e^{i \tau / 2} \operatorname{Lr} d \tau d y .
$$

Denote $\tilde{r}=\mathrm{L} r$. An explicit computation shows that $\tilde{r}$ solves

$$
i \partial_{\tau} \tilde{r}-\frac{1}{2} \mathcal{H} \tilde{r}+\frac{1}{\pi^{\frac{5}{4}}} e^{-\frac{i}{2} \tau} e^{-\frac{5}{2} y^{2}}=0, \quad \tilde{r}(0, y)=0 .
$$

By Duhamel's formula

$$
\tilde{r}(\tau, y)=\frac{i}{\pi^{5 / 4}} e^{-\frac{i \tau}{2} \mathcal{H}} \int_{0}^{\tau} e^{-\frac{i \sigma}{2}} e^{\frac{i \sigma}{2} \mathcal{H}}\left(e^{-\frac{5}{2} y^{2}}\right) d \sigma .
$$

Decompose

$$
e^{-\frac{5}{2} y^{2}}=\sum_{k \geq 0} \alpha_{k} h_{k}
$$

with $\left\{h_{k}\right\}$ 's as in (5.2) or (5.3), and

$$
\mathcal{H} h_{k}=\lambda_{k} h_{k} \equiv(2 k+1) h_{k} .
$$

Then the coefficients $\alpha_{k}$ 's are given by

$$
\alpha_{k}=\frac{1}{\sqrt{\pi}} \int_{-\infty}^{+\infty} e^{-\frac{5}{2} y^{2}} h_{k}(y) d y .
$$


Note that for $k$ odd, the eigenfunction $h_{k}$ is odd, and thus, the corresponding coefficient $\alpha_{k}=0$. In the end of this appendix we compute the rest of (even) coefficients using a generating function trick of Wang [33] and obtain

$$
\alpha_{2 j}=(-1)^{j} \frac{\sqrt{(2 j) !}}{3^{j} \sqrt{3} j !} .
$$

Since

$$
e^{\frac{1}{2} i \sigma \mathcal{H}}\left(e^{-\frac{5}{2} y^{2}}\right)=\alpha_{0} e^{\frac{1}{2} i \sigma} h_{0}+\sum_{k \geq 1} \alpha_{k} e^{i\left(k+\frac{1}{2}\right) \sigma} h_{k},
$$

by (B.2) we have

$$
\tilde{r}(\tau, y)=\frac{i}{\pi^{5 / 4}} e^{-i \frac{\tau}{2}}\left(\tau \alpha_{0} h_{0}(y)-i \sum_{k \geq 1} \frac{\alpha_{k}}{k}\left(1-e^{-i k \tau}\right) h_{k}(y)\right) .
$$

Substituting $\tilde{r}$ back into (B.1), we obtain that the zeroth term from (B.5) vanishes when integrating in $\tau$, and thus,

$$
\begin{aligned}
D_{1} & =\frac{6}{\pi^{\frac{5}{2}}} \operatorname{Re} \iint_{-\frac{\pi}{2}}^{\frac{\pi}{2}} e^{-\frac{5}{2} y^{2}} \sum_{k \geq 1} \frac{\alpha_{k}}{k}\left(1-e^{-i k \tau}\right) h_{k}(y) d \tau d y \\
& =\frac{6}{\pi^{\frac{5}{2}}} \sum_{k \geq 1} \frac{\alpha_{k}}{k} \operatorname{Re} \int_{-\frac{\pi}{2}}^{\frac{\pi}{2}}\left(1-e^{-i k \tau}\right) d \tau \int e^{-\frac{5}{2} y^{2}} h_{k}(y) d y \\
& =\frac{6}{\pi^{\frac{5}{2}}} \sum_{k \geq 1} \frac{\alpha_{k}}{k} \cdot \pi \cdot \sqrt{\pi} \alpha_{k},
\end{aligned}
$$

where we have used $\int_{-\pi / 2}^{\pi / 2} e^{-i k \tau} d \tau=0$ if $k$ is even, and $\alpha_{k}=0$ if $k$ is odd. By (B.4) and keeping only even terms $(k=2 j)$, we have

$$
D_{1}=\frac{6}{\pi} \sum_{j \geq 1} \frac{\left(\alpha_{2 j}\right)^{2}}{2 j}=\frac{1}{\pi} \sum_{j \geq 1} \frac{(2 j) !}{j 3^{2 j}(j !)^{2}},
$$

and since $\sum_{j \geq 1} \frac{(2 j) !}{j 9^{j}(j !)^{2}} \approx 0.2724$, we get

$$
D_{1}=\frac{1}{\pi} \sum_{k \geq 1} \frac{(2 k) !}{k 9^{k}(k !)^{2}} \approx \frac{1}{\pi} 0.2724 \approx 0.0867 .
$$

Proof of (B.4). Here we compute coefficients of decomposition of $e^{-\frac{5}{2} x^{2}}$ in Hermite basis, adapting a method from [33]. Recall the $k$-th Hermite polynomial $H_{k}$

$$
h_{k}(x)=\frac{H_{k}(x)}{\sqrt{2^{k} k !}} e^{-\frac{x^{2}}{2}} .
$$


We have

$$
\alpha_{k}=\frac{1}{\sqrt{2^{k} k ! \pi}} \int_{-\infty}^{+\infty} H_{k}(x) e^{-3 x^{2}} d x .
$$

Using the generating function representation

$$
e^{2 t x-t^{2}}=\sum_{n=0}^{+\infty} \frac{t^{n}}{n !} H_{n}(x)
$$

we observe that it is equivalent to

$$
e^{2 \frac{t}{\sqrt{3}} \sqrt{3} x-\left(\frac{t}{\sqrt{3}}\right)^{2}} \times e^{-\frac{2}{3} t^{2}}=\sum_{n=0}^{+\infty} \frac{t^{n}}{n !} H_{n}(x),
$$

on the other hand, using (B.8) again on the left side

$$
\sum_{j=0}^{+\infty} \frac{1}{j !}\left(\frac{t}{\sqrt{3}}\right)^{j} H_{j}(\sqrt{3} x) \times \sum_{k=0}^{+\infty} \frac{1}{k !}\left(-\frac{2}{3}\right)^{k} t^{2 k}=\sum_{n=0}^{+\infty} \frac{t^{n}}{n !} H_{n}(x) .
$$

Expanding the product on the left-hand side and identifying the powers of $t$, we get

$$
\begin{aligned}
\frac{1}{n !} H_{n}(x) & =\sum_{j+2 k=n}\left(-\frac{2}{3}\right)^{k} \frac{1}{j ! k !(\sqrt{3})^{j}} H_{j}(\sqrt{3} x) \\
& =\frac{1}{(\sqrt{3})^{n}} \sum_{j+2 k=n} \frac{(-2)^{k}}{j ! k !} H_{j}(\sqrt{3} x)
\end{aligned}
$$

Integrating both sides against $e^{-3 x^{2}}$, we obtain

$$
\begin{aligned}
\frac{1}{n !} \int H_{n}(x) e^{-3 x^{2}} d x & =\frac{1}{(\sqrt{3})^{n}} \sum_{j+2 k=n} \frac{(-2)^{k}}{j ! k !} \int_{\mathbb{R}} H_{j}(\sqrt{3} x) e^{-3 x^{2}} d x \\
& =\frac{1}{(\sqrt{3})^{n}} \sum_{j+2 k=n} \frac{(-2)^{k}}{j ! k !} \frac{1}{\sqrt{3}} \int_{\mathbb{R}} H_{0}(y) H_{j}(y) e^{-y^{2}} d y
\end{aligned}
$$

Thus by (5.4)

$$
\begin{aligned}
\frac{1}{n !} \int H_{n}(x) e^{-3 x^{2}} d x & =\frac{1}{(\sqrt{3})^{n+1}} \sum_{j+2 k=n} \frac{(-2)^{k}}{j ! k !} \sqrt{2^{j} j !} \delta_{0 j} \sqrt{\pi} \\
& = \begin{cases}0 & \text { if } n \text { is odd; } \\
\frac{(-2)^{k} \sqrt{\pi}}{(\sqrt{3})^{2 k+1} k !} & \text { if } n \text { is even, } n=2 k .\end{cases}
\end{aligned}
$$


Thus,

$$
\int_{-\infty}^{\infty} H_{2 k}(x) e^{-3 x^{2}} d x=\frac{(2 k) !}{k !} \frac{(-2)^{k} \sqrt{\pi}}{(\sqrt{3})^{2 k+1}},
$$

which by (B.7) implies that

$$
\alpha_{2 k}=(-1)^{k} \frac{\sqrt{(2 k) !}}{3^{k} \sqrt{3} k !}
$$

\section{Constant in 2D}

\section{Claim C.1.}

$$
D_{2}=\frac{1}{2 \pi} \ln \frac{4}{3}
$$

Proof. Recall from (3.18) the definition of $r$. By (3.16) we must show

$$
\operatorname{Re} \iint|G|^{2} \bar{G} r d t d x=\frac{1}{8 \pi} \ln \frac{4}{3} .
$$

We will prove this result by direct computation of the integral, which is essentially an integral of a Gaussian function (in $x$ ) and rational functions (in $s$ and $t$ ).

By (1.6),

$$
r(t, x)=\frac{i}{\pi^{3 / 2}} \int_{0}^{t} \frac{1}{(1+i s)\left(1+s^{2}\right)} e^{i \frac{(t-s)}{2} \Delta}\left(e^{-\frac{|x|^{2}}{2} \frac{(3-i s)}{\left(1+s^{2}\right)}}\right) d s
$$

Noting that

$$
e^{i \frac{t}{2} \Delta}\left(e^{-\alpha|x|^{2}}\right)=\frac{1}{(1+2 \alpha i t)^{N / 2}} e^{-\frac{\alpha|x|^{2}}{1+2 \alpha i t}}, \quad \operatorname{Re} \alpha>0
$$

we get

$$
r(t, x)=\frac{i}{\pi^{3 / 2}} \int_{0}^{t} \frac{1}{(1+i s)\left(1+s^{2}+(s+3 i)(t-s)\right)} e^{-\frac{|x|^{2}}{2} \frac{3-i s}{1+s^{2}+(s+3 i)(t-s)}} d s .
$$

Let

$$
\begin{aligned}
& A=1+s^{2}+(s+3 i)(t-s)=1+s t+3 i(t-s), \\
& B=\frac{1}{2}\left(\frac{2}{1+t^{2}}+\frac{1}{1-i t}+\frac{3-i s}{A}\right) .
\end{aligned}
$$

Thus

$$
|G|^{2} \bar{G} r=\frac{i}{\pi^{3}} \int_{0}^{t} \frac{1}{\left(1+t^{2}\right)(1-i t)(1+i s) A} e^{-|x|^{2} B} d s .
$$


Integrating in space we obtain

$$
\begin{aligned}
\int_{\mathbb{R}^{2}}|G|^{2} \bar{G} r d x & =\frac{i}{\pi^{2}} \int_{0}^{t} \frac{1}{\left(1+t^{2}\right)(1-i t)(1+i s) A B} d s \\
& =\frac{1}{\pi^{2}} \int_{0}^{t} \frac{i}{(1-i t)(1+i s)(3(1+s t)+5 i(t-s))} d s .
\end{aligned}
$$

By fraction decomposition with respect to the variable $s$,

$$
\begin{aligned}
\operatorname{Re} & {\left[\frac{i}{(1-i t)(1+i s)(3(1+s t)+5 i(t-s))}\right] } \\
& =\operatorname{Re}\left[\frac{1}{8\left(1+t^{2}\right)}\left(\frac{i}{1+i s}+\frac{5 i-3 t}{3+5 i t+(3 t-5 i) s}\right)\right] \\
& =\frac{1}{8\left(1+t^{2}\right)}\left(\frac{s}{1+s^{2}}+\frac{25(t-s)-9 t(1+t s)}{9(1+t s)^{2}+25(t-s)^{2}}\right) .
\end{aligned}
$$

Integrating with respect to the variable $s$ and coming back to (C.1) we get:

$$
\operatorname{Re}\left(\int_{\mathbb{R}^{2}}|G|^{2} \bar{G} r d x\right)=-\frac{1}{16 \pi^{2}} \frac{\ln \left(1+t^{2}\right)+2 \ln 3-\ln \left(9+25 t^{2}\right)}{1+t^{2}} .
$$

Finally, we compute the space-time norm:

$$
\begin{aligned}
& \int_{-\infty}^{\infty} \operatorname{Re}\left(\int_{\mathbb{R}^{2}}|G|^{2} \bar{G} r d x\right) d t \\
& =-\frac{1}{16 \pi^{2}}\left(\int_{-\infty}^{\infty} \frac{\ln \left(1+t^{2}\right)}{1+t^{2}} d t+2 \ln 3 \int_{-\infty}^{\infty} \frac{d t}{1+t^{2}}-\int_{-\infty}^{\infty} \frac{\ln \left(9+25 t^{2}\right)}{\left(1+t^{2}\right)} d t\right) .
\end{aligned}
$$

We have

$$
\int_{-\infty}^{\infty} \frac{1}{\left(1+t^{2}\right)} d t=\pi
$$

By the change of variable $t=\tan \tau, \tau \in(-\pi / 2, \pi / 2)$ and the classical formulas

$$
\begin{aligned}
& \int_{0}^{\frac{\pi}{2}} \ln (\cos \tau) d \tau=-\frac{\pi}{2} \ln 2 \\
& \int_{0}^{\pi} \ln (a+b \cos \tau) d \tau=\pi \ln \left(\frac{a+\sqrt{a^{2}-b^{2}}}{2}\right), a>|b|
\end{aligned}
$$

one gets

$$
\int_{-\infty}^{\infty} \frac{\ln \left(9+25 t^{2}\right)}{\left(1+t^{2}\right)}=6 \pi \ln 2, \quad \int_{-\infty}^{\infty} \frac{\ln \left(1+t^{2}\right)}{\left(1+t^{2}\right)} d t=2 \pi \ln 2
$$


We leave the details of the computations to the reader. Combining the preceding equalities, we obtain as announced

$$
\int_{-\infty}^{\infty} \operatorname{Re}\left(\int_{\mathbb{R}^{2}}|G|^{2} \bar{G} r d x\right) d t=\frac{1}{8 \pi}(\ln 4-\ln 3) .
$$

\section{Bound of a sum of binomial coefficients}

Let $m \geq 1$ and $j \in\{0, \ldots, m\}$. In this appendix we sketch the proof of the following inequality

$$
\sum_{\substack{k \in\{0, \ldots, m\} \\
j+k \text { even }}}\left(\begin{array}{c}
j+k \\
j
\end{array}\right)\left(\begin{array}{c}
2 m-(j+k) \\
m-j
\end{array}\right) \leq \frac{1}{2}\left(\begin{array}{c}
2 m+1 \\
m+1
\end{array}\right)+\frac{1}{2}\left(\begin{array}{c}
2 m \\
m
\end{array}\right) .
$$

For $n \in \mathbb{N}^{*}$, let $I_{n}=\{1, \ldots, n\}$. Let $\mathcal{P}\left(I_{2 m+1}\right)$ be the set of all subsets of $I_{2 m+1}$. Define $O_{m, j} \subset \mathcal{P}\left(I_{2 m+1}\right)$ and $E_{m, j} \subset \mathcal{P}\left(I_{2 m+1}\right)$ as follows: a subset of $I_{2 m+1}$ is in $O_{m, j}$ (respectively, $E_{m, j}$ ) if it has $m+1$ elements $a_{1}<a_{2}<\ldots<a_{m+1}$ and if $a_{j+1}$ is odd (respectively, even). Then for fixed $j \in\{0, \ldots, m\}$,

$$
\left|O_{m, j}\right|=\sum_{\substack{k \in\{0, \ldots, m\} \\
j+k \text { even }}}\left(\begin{array}{c}
j+k \\
j
\end{array}\right)\left(\begin{array}{c}
2 m-(j+k) \\
m-j
\end{array}\right), \quad\left(\begin{array}{c}
2 m+1 \\
m+1
\end{array}\right)=\left|O_{m, j}\right|+\left|E_{m, j}\right| .
$$

Let us construct a one-to-one map $\Phi_{j}$ from $O_{m, j}$ to the disjoint union of $E_{m, j}$ and the set of $m$-elements subsets of $I_{2 m}$. Let $S$ be a set which is in $O_{m, j}$, and $a_{1}<a_{2}<\ldots<a_{m+1}$ its $m+1$ elements. Then if $j \geq 1$ and $a_{j}<a_{j+1}-1$, or $j=0$ and $a_{1}>1$, we denote by $\Phi_{j}(S)$ the element of $E_{m, j}\left\{a_{1}, \ldots, a_{j}, a_{j+1}-\right.$ $\left.1, a_{j+2}, \ldots, a_{m+1}\right\}$ (i.e obtained from $S$ by shifting only the element $a_{j+1}$ to the left). If $a_{j}=a_{j+1}-1$, or $j=0$ and $a_{1}=1$, we denote by $\Phi_{j}(S)$ the subset $\left\{a_{1}, \ldots, a_{j}, a_{j+2}, \ldots, a_{m}\right\}$ of $I_{2 m}$. The mapping $\Phi_{j}$ is clearly one-to-one: in the first case one can recover $S$ by shifting the $j+1$ element of $\Phi_{j}(S)$ to the right. In the second case, by adding to the set $\Phi_{j}(S)$ the element $b_{j}+1(1$ if $j=0)$, where $b_{j}$ is the $j$ th element of $\Phi_{j}(S)$. Finally we obtain:

$$
\left|O_{m, j}\right| \leq\left|E_{m, j}\right|+\left(\begin{array}{c}
2 m \\
m
\end{array}\right) \leq\left(\begin{array}{c}
2 m+1 \\
m+1
\end{array}\right)-\left|O_{m, j}\right|+\left(\begin{array}{c}
2 m \\
m
\end{array}\right)
$$

which yields (D.1). 


\section{References}

[1] J. Bourgain. Refinements of Strichartz' inequality and applications to 2D-NLS with critical nonlinearity, Internat. Math. Res. Notices (1998), 253-283.

[2] J. Bourgain, Global wellposedness of defocusing critical nonlinear Schrödinger equation in the radial case, J. Amer. Math. Soc. 12 (1999), 145-171.

[3] P. BÉGOUT and A. VARGAS, Mass concentration phenomena for the $L^{2}$-critical nonlinear Schrödinger equation, Trans. Amer. Math. Soc. 359 (2007), 5257-5282.

[4] R. CARLES, Critical nonlinear Schrödinger equations with and without harmonic potential, Math. Models Methods Appl. Sci. 12 (2002), 1513-1523.

[5] R. CARLES, Rotating points for the conformal nls scattering operator, Dyn. Partial Differ. Equ. 6 (2009), 35-51.

[6] J. Colliander, M. Keel, G. Staffilani, H. Takaoka and T. TaO, Global wellposedness and scattering for the energy-critical nonlinear Schrödinger equation in $\mathbb{R}^{3}$, Ann. of Math. 167 (2008), 767-865.

[7] P. Constantin and J.-C. SAUT, Local smoothing properties of Schrödinger equations, Indiana Univ. Math. J. 38 (1989), 791-810.

[8] T. CAZENAVE and F. B. WeISSleR, The Cauchy problem for the critical nonlinear Schrödinger equation in $H^{s}$, Nonlinear Anal. 14 (1990), 807-836.

[9] T. DUYCKAERTS and F. MERLE, Scattering norm estimate near the threshold for energycritical focusing semilinear wave equation, Indiana Univ. Math. J. 58 (2009), 1971-2002.

[10] K.-J. ENGEL and R. NAGEL, "One-parameter Semigroups for Linear Evolution Equations", Vol. 194 of Graduate Texts in Mathematics, Springer-Verlag, New York, 2000, with contributions by S. Brendle, M. Campiti, T. Hahn, G. Metafune, G. Nickel, D. Pallara, C. Perazzoli, A. Rhandi, S. Romanelli and R. Schnaubelt.

[11] D. FosCHI, Maximizers for the Strichartz inequality, J. Eur. Math. Soc. (JEMS) 9 (2007), $739-774$.

[12] J. HolmeR and S. RoudenKo, A sharp condition for scattering of the radial $3 D$ cubic nonlinear Schrödinger equation, Comm. Math. Phys. 282 (2008), 435-467.

[13] D. Hundertmark and V. Zharnitsky, On sharp Strichartz inequalities in low dimensions, Int. Math. Res. Not. 2006, Art. ID 3408, 18 pages.

[14] S. KERAANI, On the blow up phenomenon of the critical nonlinear Schrödinger equation, J. Funct. Anal. 235 (2006), 171-192.

[15] C. E. KenIG and F. MERLE, Global well-posedness, scattering and blow-up for the energycritical, focusing, non-linear Schrödinger equation in the radial case, Invent. Math. 166 (2006), 645-675.

[16] R. KILliP, T. TAO and M. VISAN, The cubic nonlinear schrödinger equation in two dimensions with radial data, J. Eur. Math. Soc. (JEMS) 11 (2009), 1203 -1258.

[17] P.-L. LIONS, The concentration-compactness principle in the calculus of variations. The locally compact case, I, Ann. Inst. H. Poincaré Anal. Non Linéaire 1 (1984), 109-145.

[18] P.-L. LIONS, The concentration-compactness principle in the calculus of variations. The limit case, I, Rev. Mat. Iberoamericana 1 (1985), 145-201.

[19] F. Merle and L. Vega, Compactness at blow-up time for $L^{2}$ solutions of the critical nonlinear Schrödinger equation in 2D, Internat. Math. Res. Notices (1998), 399-425.

[20] U. NIEDERER, The maximal kinematical invariance groups of Schrödinger equations with arbitrary potentials, Helv. Phys. Acta 47 (1974), 167-172.

[21] O. REY, The role of the Green's function in a nonlinear elliptic equation involving the critical Sobolev exponent, J. Funct. Anal. 89 (1990), 1-52.

[22] A. V. Rybin, G. G. Varzugin, M. Lindberg, J. Timonen and R. K. Bullough, Similarity solutions and collapse in the attractive gross-pitaevskii equation, Phys. Rev. E 62 (2000), 6224-6228.

[23] P. SJöLIN, Convergence properties for the Schrödinger equation, Rend. Sem. Mat. Fis. Milano 57 (1989), 293-297. 
[24] R. S. STRICHARTZ, Restrictions of Fourier transforms to quadratic surfaces and decay of solutions of wave equations, Duke Math. J. 44 (1977), 705-714.

[25] V.I. TALANOV, Focusing of light in cubic media, JETP Lett. 11, 199-201.

[26] T. TAO, Global well-posedness and scattering for the higher-dimensional energy-critical nonlinear Schrödinger equation for radial data, New York J. Math. 11 (2005), 57-80 (electronic).

[27] T. TAO, Spacetime bounds for the energy-critical nonlinear wave equation in three spatial dimensions, Dyn. Partial Differ. Equ. 3 (2006), 93-110.

[28] T. TAO, A pseudoconformal compactification of the nonlinear Schrödinger equation and applications, New York J. Math. 15 (2009), 265-282.

[29] T. TAO and M. VISAN, Stability of energy-critical nonlinear Schrödinger equations in high dimensions, Electron. J. Differential Equations (2005), pages No. 118, 28 pp. (electronic).

[30] T. TAO, M. VISAN and X. ZHANG, Global well-posedness and scattering for the defocusing mass-critical nonlinear Schrödinger equation for radial data in high dimensions, Duke Math. J. 140 (2007), 165-202.

[31] T. TAO, M. VISAN and X. ZHANG, Minimal-mass blowup solutions of the mass-critical NLS, Forum Math. 20 (2008), 881-919.

[32] L. VeGA, Schrödinger equations: pointwise convergence to the initial data, Proc. Amer. Math. Soc. 102 (1988), 874-878.

[33] W.-M. WANG, Pure point spectrum of the Floquet Hamiltonian for the quantum harmonic oscillator under time quasi-periodic perturbations, Comm. Math. Phys. 277 (2008), 459496.

Département de Mathématiques

Université de Cergy-Pontoise

Site de Saint Martin

2, avenue Adolphe Chauvin

95302 Cergy-Pontoise Cedex, France

thomas.duyckaerts@u-cergy.fr

frank.merle@u-cergy.fr

Department of Mathematics

2115 G. Street NW

The George Washington University

Washington, DC 20052, USA

roudenko@gwu.edu 